Sun et al.

Identification of changing ribosomes using cryo-EM and MS

EMBL ver $2.8(02 / 12 / 2018)$

\title{
Identification of changing ribosome protein compositions using cryo-EM and
}

\section{mass spectrometry}

Ming Sun ${ }^{1 *}$, Parimal Samir ${ }^{2,3^{*}}$, Bingxin Shen ${ }^{4 *}$, Wen $\mathrm{Li}^{4}$, Christopher M. Browne ${ }^{2}$, Rahul ${ }^{5}$, Joachim Frank $^{1,4 \#}$ and Andrew J. Link ${ }^{2,3 \#}$

${ }^{1}$ Department of Biological Sciences, Columbia University, New York, NY, 10027, U.S.A.

${ }^{2}$ Department of Biochemistry, Vanderbilt University School of Medicine, Nashville, TN, 37235, U.S.A.

${ }^{3}$ Department of Pathology, Microbiology and Immunology, Vanderbilt University School of Medicine, Nashville, TN, 37235, U.S.A.

${ }^{4}$ Department of Biochemistry and Molecular Biophysics, Columbia University, New York, 10032, U.S.A.

${ }^{5}$ University of Waterloo, Waterloo, ON, Canada

*Equal contributions

\# Corresponding authors

Andrew J. Link

Dept. of Pathology, Microbiology and Immunology

Vanderbilt University School of Medicine

$116121^{\text {st }}$ Ave South

Nashville, TN 37232

TEL: 615-343-6823

FAX: 615-343-7392

andrew.link@vanderbilt.edu

Joachim Frank

Department of Biochemistry and Molecular Biophysics

Columbia University

650 W. $168^{\text {th }}$ Street

New York, NY 10032

jf2192@cumc.columbia.edu 
Sun et al.

Identification of changing ribosomes using cryo-EM and MS

EMBL ver $2.8(02 / 12 / 2018)$

\section{Summary}

The regulatory role of the ribosome in gene expression has come into sharper focus. It has been proposed that ribosomes are dynamic complexes capable of changing their protein composition in response to enviromental stimuli. We applied both cryo-EM and mass spectrometry to identify such changes in S. cerevisiae $80 \mathrm{~S}$ ribosomes. Cryo-EM shows a fraction (17\%) of the ribosome population in yeast growing in glucose lack the ribosomal proteins RPL10 (ul16) and RPS1A/B (eS1). Unexpectedly, this fraction rapidly increases to $34 \%$ after the yeast are switched to growth in glycerol. Using quantitative mass spectrometry, we found that the paralog yeast ribosomal proteins RPL8A (eL8A) and RPL8B (eL8B) change their relative proportions in the $80 \mathrm{~S}$ ribosome when yeast are switched from growth in glucose to glycerol. Using yeast genetics and polysome profiling, we show that yeast ribosomes containing either RPL8A or RPL8B are not functionally interchangeable. Our combined cryo-EM and quantitative proteomic data support the hypothesis that ribosomes are dynamic complexes that alter their composition and functional activity in response to changes in growth or environmental conditions.

\section{Keywords}

Ribosome heterogeneity, carbon source switch, ribosome filter, RPS1A/B, RPL10, RPL8A/B

\section{Highlights}

- A fraction of ribosomes in yeast cells growing in glucose lacks two essential proteins and appears inactive.

- This inactive fraction doubles when the cells are switched from glucose to glycerol as the carbon source.

- The switch in carbon source induces compositional changes in ribosomal protein paralogs in the ribosomes and functional changes based on polysome profiles. 
bioRxiv preprint doi: https://doi.org/10.1101/271833; this version posted February 26, 2018. The copyright holder for this preprint (which was not certified by peer review) is the author/funder, who has granted bioRxiv a license to display the preprint in perpetuity. It is made available under aCC-BY-NC-ND 4.0 International license.

Sun et al.

Identification of changing ribosomes using cryo-EM and MS

EMBL ver $2.8(02 / 12 / 2018)$

- RPL8A and RPLB change their relative stoichiometry in the ribosome when yeast cells are grown in different carbon sources. 
Sun et al.

Identification of changing ribosomes using cryo-EM and MS

EMBL ver $2.8(02 / 12 / 2018)$

\section{Introduction}

Gene expression can be regulated at multiple levels, including transcription and translation. Translation is the process by which the information encoded in an mRNA is used to synthesize polypeptides. Composed of a small $40 \mathrm{~S}$ and large $60 \mathrm{~S}$ subunit, the eukaryotic $80 \mathrm{~S}$ ribosome catalyzes the decoding of mRNAs and formation of peptide bonds. Translational control is a major mechanism modulating eukaryotic gene expression (Carpenter et al., 2014; Costa-Mattioli et al., 2009; Hinnebusch, 2015; Holcik and Sonenberg, 2005; Kong and Lasko, 2012). Numerous studies have shown that a large number of translation factors, posttranslational protein modifications, and RNA accessory factors play active roles in translational control (Dever and Green, 2012; Hinnebusch, 2015; Kapp and Lorsch, 2004; Sonenberg and Hinnebusch, 2009). Initially, the ribosomes themselves were considered only passive players in this process (Mauro and Edelman, 2002).

In recent years, the idea that the ribosomes can function as transcript-specific posttranscriptional regulatory elements has been formulated (Komili et al., 2007; Kondrashov et al., 2011; McIntosh and Warner, 2007; Ruggero and Pandolfi, 2003; Warner, 2015; Warner and McIntosh, 2009; Xue et al., 2015). In the depot model, ribosomes act as reservoirs of regulatory molecules, which are released in response to specific cellular cues (Mazumder et al., 2003; Ray et al., 2007; Zhou et al., 2015). An example of this mode of action is the role of the human RPL13A ribosomal protein (r-protein) in translational control during the inflammatory response (Kapasi et al., 2007; Mazumder et al., 2003). A second model proposes a more direct role of ribosomes in translational control (Mauro and Edelman, 2002; Mauro and Edelman, 2007). This model derives from studies that reveal ribosomes are not the static, uniform structures described in textbooks. Comparison of ribosomes from rat skeletal muscle and liver using 2-D gel electrophoresis revealed differences in ribosomal composition between the two tissues (Sherton and Wool, 1974). In the amoeba Dictyostelium discoideum ribosomes from spores and vegetative cells differ both in protein composition and posttranslational modifications (Ramagopal and 
Sun et al.

Identification of changing ribosomes using cryo-EM and MS

EMBL ver 2.8 (02/12/2018)

Ennis, 1981). In the S. cerevisiae genome, the 79 r-proteins in the $80 \mathrm{~S}$ ribosome are encoded by 138 genes (Nakao et al., 2004; Table S1). These include 57 duplicate, paralogous ribosomal gene pairs (McIntosh and Warner, 2007; Warner, 1999; Table S1), raising the possibility that individual paralogs have distinct finctions. Deletion analysis of S. cerevisiae paralogous pairs showed that the genes are not functionally equivalent (Breslow et al., 2008; Giaever et al., 2002; Komili et al., 2007). Using mass spectrometry, differences were found in the stoichiometry of core r-proteins in yeast grown under different growth conditions (Slavov et al., 2015). In higher eukaryotic organisms, previous studies showed specific r-proteins are required for the translation of selected mRNA transcripts (Kondrashov et al., 2011; Lee et al., 2013). Post-translational modifications, especially phosphorylation, and variations in rRNA composition add further levels of complexity to the different ribosome complexes in cells and tissues (Martin et al., 2014; Ramagopal, 1992).

The evidence of heterogeneity in the ribosome's protein and rRNA composition has generated speculation as to its functional consequences. Mauro and Edelman proposed that ribosomal subunits differing in protein or rRNA composition bind translation factors or specific mRNAs with different affinities, thereby selectively changing the rates of polypeptide synthesis of particular mRNAs (Mauro and Edelman, 2002; Mauro and Edelman, 2007). In their model, different or specialized ribosomes in a heterogeneous ribosomal population in the cell function as regulatory elements or 'filters' by selectively binding and translating specific mRNAs. In support of this model, Bauer et al. showed that "specialized" ribosomes with specific r-proteins encoded by paralogous genes preferentially translate particular reporter mRNAs (Bauer et al., 2013).

Cells growing under one growth condition require a specific proteome, and the cells growing in a second condition require a different proteome. A number of cellular mechanisms including transcription, RNA processing, translation, and protein degradation are known to regulate gene expression and ultimately the cell's proteome when growth conditions change. In this study, we hypothesized that the 
Sun et al.

Identification of changing ribosomes using cryo-EM and MS

EMBL ver 2.8 (02/12/2018)

cell's ribosomes also change their protein composition in response to changing growth conditions as a mechanism to regulate expression. To test this hypothesis, we used a combined strategy of single-particle cryo-electron microscopy (cryo-EM) and quantitative mass spectrometry-based proteomics to detect and identify specific r-proteins that change their stoichiometry in the $80 \mathrm{~S}$ ribosome upon change of growth condition. Cryo-EM is a method for imaging molecules in their native, hydrated state at cryogenic temperature by transmission electron microscopy (Frank, 2006). When applied to the ribosome, cryo-EM can be used to reconstruct the ribosome at molecular resolution in three dimensions. Moreover, the technique allows ribosomes to be grouped into different classes, or subpopulations, based upon structural similarities and differences. Reconstructions of the individual classes can be compared with a highresolution reference structure of the intact 80 S ribosome to identify subpopulations lacking densities for $\mathrm{r}$ proteins. In this way, cryo-EM can be used to detect and identify ribosomes with sub-stoichiometric rprotein compositions. Quantitative proteomics using tandem mass spectrometry analysis of proteasedigested ribosomes coupled with isotopic labeling can be used to both identify and quantify the relative abundances of specific proteins in cellular or subcellular protein fractions (Ross et al. MCP 3, 1154, 2004; Yates et al., 2009). Thus, cryo-EM and quantitative proteomics should be able complement each other's strengths in a comprehensive approach to identify changes in 80S ribosomal protein compositions.

Using such a combined strategy, we profiled the $80 \mathrm{~S}$ ribosome composition in yeast cells growing in glucose or glycerol as a carbon source. Abruptly shifting from fermentable glucose to the nonfermentable glycerol carbon source induces a diverse array of changes in S. cerevisiae gene expression (Kuhn et al MCB, 21, 916, 2001; Samir et al., 2015). In this study, ribosomes from yeast cells growing in glucose and then glycerol were isolated using sucrose gradients and split into two pools for cyro-EM and quantitative proteomics. Cryo-EM identified a significant fraction of $80 \mathrm{~S}$ ribosomes from glucose growth lacking the essential r-protein RPL10 or both RPL10 and RPS1A/B. This fraction rapidly increased upon switching to growth in glycerol. Importantly, these classes of ribosomes also lacked 
Sun et al.

Identification of changing ribosomes using cryo-EM and MS

EMBL ver 2.8 (02/12/2018)

tRNAs, as well as any conformational variability known to be associated with active translation. Using proteomic analysis, we identified 75 of the expected 79 r-proteins in the $80 \mathrm{~S}$ complexes. This set included 55 of the 57 paralogous r-protein gene pairs. Proteomic analysis revealed that the r-protein paralogs RPL8A and RPL8B change their relative stoichiometry in the population of ribosomes when yeast cells are shifted from glucose to glycerol as a carbon source. Phenotypic analysis and polysome profiling using

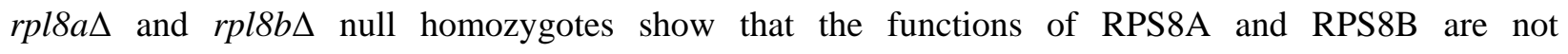
interchangeable.

\section{Results}

We hypothesized that specific r-proteins important for the specialization of ribosome activity will show differential abundances in response to environmental stimuli. In pilot studies, we first used quantitative proteomics to identify and quantify changes in individual r-proteins abundances from yeast whole-cell protein extracts after shifts in both carbon source and temperature (Samir et al., 2015). The analysis showed most of the $40 \mathrm{~S}$ and $60 \mathrm{~S}$ r-proteins were down-regulated in response to the two environmental stimuli ( $\underline{\text { Fig. 1) }}$. However, selected r-proteins showed significantly larger decreases in abundance (Samir et al., 2015). This result suggested that these r-proteins were being differentially expressed compared to the majority of the r-proteins, consistent with the ribosome filter hypothesis (Mauro and Edelman, 2002).

In order to directly measure the changes in the protein composition of $80 \mathrm{~S}$ ribosomes purified from yeast, we focused on comparing yeast growing in rich medium with two different carbon sources, glucose and glycerol (Samir et al., 2015). From yeast cells cultured in glucose or glycerol, we isolated ribosomes using discontinuous sucrose gradient centrifugation to use for both cryo-EM and quantitative proteomic analysis (Samir et al., 2015). 
Sun et al.

Identification of changing ribosomes using cryo-EM and MS

EMBL ver 2.8 (02/12/2018)

\section{Cryo-EM studies of S. cerevisiae ribosomes at multiple time points}

To study the changes in the structure and composition of $80 \mathrm{~S}$ ribosomes over time after changing from glucose to glycerol, we first performed cryo-EM experiments on $80 \mathrm{~S}$ ribosomes harvested from ribosomal samples at different time points. An aliquot of cells growing in glucose was collected $(\mathrm{T}=0 \mathrm{~min})$ and the cells were shifted to growth in glycerol. We then harvested cells at 30, 120, 240, 450 and 1440 min after shifting to glycerol and purified ribosomes using sucrose gradients. The cryo-EM study proceeded in two stages. In the initial stage, the method of classification and quantification of ribosome subpopulations was developed and validated with a dataset collected on a $200-\mathrm{kV}$ instrument using a CCD camera (Shen, B, Sun, M., Li, W., Samir, P., Link, A. and Frank, J., in preparation). In the present full-scale study, a much larger dataset was collected on a $300-\mathrm{kV}$ instrument using a direct electron detection camera.

Initial cryo-EM study. Initial cryo-EM experiments were performed on the purified yeast $80 \mathrm{~S}$ ribosomes after the shift from glucose to glycerol, using an FEI Tecnai F20 electron microscope (FEI, Portland, OR) at $200 \mathrm{kV}$, equipped with a 4k x 4k CCD camera (Gatan, Pleasanton, CA). A total of $\sim 121,000$ particles were selected from 2,661 micrographs from the six time-resolved sample sets. Hierarchical classification was performed using the RELION program (Scheres, 2012), in conjunction with a quantitative analysis of convergence (Chen et al., 2014). To quantitatively analyze the results, we used a pooled classification scheme that tracks the shifts of sub-populations and ensures the maximum number of particles for the reconstruction of each ribosome class (Shen, B, Sun, M., Li, W., Samir, P., Link, A. and Frank, J., in preparation). The majority of the cryo-EM ribosome images at $\mathrm{T}=0$ were of complete $80 \mathrm{~S}$ ribosomes, bound with P/E-tRNA, A/P-P/E-tRNAs or P-E-tRNAs, strongly suggesting they were biologically functional. However, a fraction ( 20\%) lacked the density for r-proteins RPS1A/B (eS1) and RPL10 (uL16) and showed no tRNA. Unexpectedly, 30 min after shifting from glucose to glycerol, the fraction of $80 \mathrm{~S}$ ribosomes lacking these r-proteins increased considerably (to $~ 32 \%$ ) and remained at this level. 
Sun et al.

Identification of changing ribosomes using cryo-EM and MS

EMBL ver 2.8 (02/12/2018)

Full-scale cryo-EM study. Prompted by the observation of the change in r-protein composition of the 80S ribosomes, we used an FEI Polara TF30 electron microscope operating at 300kV, equipped with GATAN K2 Summit direct detector camera (Gatan, Pleasanton, CA), to collect a larger dataset in an attempt to improve the resolution. A total number of $~ 291,000$ particles were selected from 6,576 micrographs from the six sample sets (i.e., glucose at $\mathrm{T}=0$ and five different time points after the switch to growth in glycerol). The resulting six cryo-EM data sets were pooled together for further classification and refinement, following the protocol established in the initial study (Shen, B, Sun, M., Li, W., Samir, P., Link, A. and Frank, J., in preparation; for an overview of the data processing procedure, see Fig. S1). The first tier in the hierarchical unsupervised classification revealed two major ribosomal populations, complete $80 \mathrm{~S}$ ribosomes and incomplete $80 \mathrm{~S}$ ribosomes lacking densities for r-proteins RPS1A/B (eS1) and/or RPL10 (uL16).

Since reconstructions from both populations showed fragmented densities, we performed a second run of exhaustive unsupervised classification. For the population of complete $80 \mathrm{~S}$ ribosomes, we identified five major subpopulations differing in conformation and occupancy by tRNAs (Fig. S1 and Table S2). We also identified two types of conformational changes, inter-subunit rotation and small subunit head swivel, which are known to accompany mRNA-tRNA translocation. The findings of tRNAs and their associated conformational changes is evidence of actively translating ribosomes (Frank and Gonzalez, 2010). For brevity, 80S ribosomes with and without evidence of inter-subunit rotation will be termed "rotated" and "non-rotated," respectively. Three (i-iii) of the five subpopulations showed rotated 80S ribosomes bound with hybrid P/E tRNA (denoted as rt80-P/E), differing in the extent of their 40S subunit head swivel. One (iv) showed non-rotated $80 \mathrm{~S}$ ribosomes bound with classically configured Pand E-tRNAs (denoted as nrt80S-P/E); and one (v) showed non-rotated 80S without tRNAs (denoted as nrt80S-empty). The resolutions of the density maps reconstructed from these subclasses ranged from $5.6 \AA$ to $7.6 \AA$ (here and henceforth, all resolutions were measured by $\mathrm{FSC}=0.143$, following the 'gold-standard' 
Sun et al.

Identification of changing ribosomes using cryo-EM and MS

EMBL ver 2.8 (02/12/2018)

protocol (Chen et al., 2013; Scheres, 2012)). Thus, with the exception of the least common subclass (v), the class of complete ribosomes with a complete set of r-proteins displays tRNA occupancies and conformational variability compatible with active translational activity.

For the population of incomplete $80 \mathrm{~S}$ ribosomes identified in the first classification step, further subclassification revealed five different subpopulations, distinguished by the presence versus absence of one or two r-proteins as well as conformational changes (Fig. S1 and S2 and Table S3): (a) 80S lacking density for RPL10 (uL16) (ig. 2A); (b-c) 80S lacking densities for both RPL10 (uL16) and RPS1A/B (eS1) (Fig. 2B and C); (d-e) 80S lacking densities for both RPL10 (uL16) and RPS1A/B (eS1), as well as densities for small fragments of rRNA (Fig. 2D and E). The resolutions of these density maps range from $6.2 \AA$ to $9.8 \AA$.

\section{Locations and interacting partners of proteins uL16 and eS1}

The most notable finding in our cryo-EM study is that upon carbon source switch from glucose to glycerol, the fraction of the $80 \mathrm{~S}$ ribosome particles missing the r-proteins RPS1A/B (eS1) (from 40S subunits) and RPL10 (uL16) (from 60S subunits) increases rapidly. The small-subunit r-protein RPS1A/B (eS1) is located in the vicinity of the mRNA exit tunnel and has direct contacts with helix 26 (H26) of the 18S rRNA and r-proteins RPS14A/B (uS11) and RPS26A/B (eS26). In the class of incomplete 80S ribosomes lacking RPS1A/B (eS1), we also found that RPS14A/B (uS11) and RPS26A/B (eS26) either displayed scattered densities or were completely absent from the ribosome, suggesting weak binding affinities of these proteins to 40S subunits lacking RPS1A/B (eS1) (i․ 2).

The large-subunit protein RPL10 (uL16) is located in the inter-subunit corridor through which tRNAs move during the peptide elongation cycle. It has contacts with several key, functionally important sites via its C-terminal region (P-loop), including the elongation factor binding site, the peptidyltransferase center (PTC), and H38 of the 28S rRNA (also known as the A-site finger). Protein RPL10 (uL16) also has interactions with the 5S rRNA via its C-terminus and with H69 of the 28S rRNA via its 
Sun et al.

Identification of changing ribosomes using cryo-EM and MS

EMBL ver $2.8(02 / 12 / 2018)$

conserved internal loop (Sulima et al., 2014). In the population of incomplete 80 S ribosomes, we found that H38 and its neighboring helices display only scattered densities (i․ 2D and 2E and Table S33), indicating variable positions. Such positional variability is not commonly observed in mature $80 \mathrm{~S}$ ribosome structures but has been found in another, independent study of $60 \mathrm{~S}$ subunit biogenesis (Malyutin et al., 2017).

\section{The binding affinity of eS1 and uL16 to $80 \mathrm{~S}$ ribosomes changes upon switch of carbon source}

To quantitatively investigate the binding behavior of RPS1A/B (eS1) and RPL10 (uL16) to ribosomes, we monitored the number of ribosomal particles that were assigned to the populations of 'complete $80 \mathrm{~S}$ ' and 'incomplete $80 \mathrm{~S}$ ' at different time points $(\mathrm{T}=0,30,120,240,450$, and $1440 \mathrm{~min}$ after changing growth in glucose to glycerol) using our pooled classification strategy. In order to test experimental reproducibility, we performed the same pooling and tracking strategy in two independent cryo-EM experiments. In both studies, the quantity of particles assigned to the 'incomplete $80 \mathrm{~S}$ ' population increased in the first $30 \mathrm{~min}$ after shifting from glucose to glycerol, as the binding ratio of RPL10 (uL16) and RPS1A/B (eS1) to 80S ribosomes decreased from $\sim 83 \%$ to $\sim 66 \%$, and then remained relatively stable: $\sim 64 \%$ at $\mathrm{T}=120 \mathrm{~min}$ and $\sim 62 \%$ at $\mathrm{T}=240 \mathrm{~min}(\underline{\text { Fig. 3) }}$. After incubation in glycerol for 450 min, the binding of RPL10 (uL16) and RPS1A/B (eS1) to $80 \mathrm{~S}$ ribosomes recovered from $62 \%$ to $\sim 71 \%$ at $\mathrm{T}=450 \mathrm{~min}$ and stabilized at $\sim 73 \%$ at $\mathrm{T}=1440 \mathrm{~min}(24 \mathrm{~h})$ (Fig. 3).

\section{Quantitative proteomic analysis of r-proteins in purified ribosomes.}

To identify and measure the changes in the protein composition of the same ribosome populations purified from yeast growing in glucose or glycerol, we analyzed the ribosomal protein composition using iTRAQ-labeling and quantitative tandem mass spectrometry (Link et al., 1999; Ross et al., 2004). Whole-cell extract and purified ribosome sample from yeast cells grown in glucose $(\mathrm{T}=0)$ and the $24 \mathrm{~h}$ glycerol $(\mathrm{T}=24 \mathrm{~h})$ time points were used. After proteolytic digestion of the proteins with trypsin, the Ntermini and amino acid side chain amines of the tryptic fragments were covalently labeled with isotoptic 
Sun et al.

Identification of changing ribosomes using cryo-EM and MS

EMBL ver 2.8 (02/12/2018)

4-plex mass tags (Ross et al., 2004; Unwin et al., 2010). After replicate ribosome samples from the glucose $(\mathrm{T}=0)$ and the $24 \mathrm{~h}$ glycerol $(\mathrm{T}=24 \mathrm{~h})$ time points were labeled with unique iTRAQ tags, we pooled the iTRAQ-labeled ribosome samples and fractionated the peptide mixture using multidimensional microcapillary HPLC (Link et al., 1999). The eluting peptides were analyzed using nanoESItandem mass spectrometry (MS/MS)(Samir et al., 2015). The peptide fragmentation data were computationally compared to predicted values from a yeast protein database to identify the labeled peptides and hence the corresponding proteins (Eng et al., 1994). The fragmentation of the iTRAQ tag generated low molecular mass reporter ions that were used to quantify the identified r-protein peptides and proteins (Ross $e t$ al., 2004; Unwin et al., 2010).

From three independent biological replicates, we identified 135 of the 139 r-proteins from the purified ribosome complexes using mass spectrometry-based proteomic ( $\underline{\text { Table S4}})$. Of the 79 r-yeast proteins in the $80 \mathrm{~S}$ ribosomal complexes, we successfully identified 20 of the 22 r-proteins from singlecopy genes and 55 of the 57 r-protein pairs from paralogous genes. The four proteins not identified were from r-protein genes encoding small proteins $<60$ amino acids in size. The mass spectrometry analysis identified 21 of the $22100 \%$ identical paralogous r-proteins (able S4). For 16 of the 35 paralogous gene pairs that are not $100 \%$ identical, we successfully identified both unique peptides. Of the remaining 19 non-identical paralogous gene pairs, our mass spectrometry analysis was able to uniquely identify 1 of the 2 paralogous r-proteins in 8 cases ( $\underline{\text { Table } \mathbf{S 4}}$ ).

For each of the three replicates, we next calculated the relative fold-change in abundance for 131 r-proteins in glycerol $(\mathrm{T}=24)$ compared to glucose $(\mathrm{T}=0)$ using the $\log _{2}$ ratio of the iTRAQ reporter ion signal intensities (Table S5). Because ribosomes were previously purified and analyzed from identical whole-cell extracts (Samir et al., 2015), we were able to directly compare the fold changes of 126 rproteins quantitated in both the whole-cell extracts and purified ribosomes. A correlation analysis between the fold change of r-proteins in the solubilized whole cell extracts and the purified ribosome 
Sun et al.

Identification of changing ribosomes using cryo-EM and MS

EMBL ver 2.8 (02/12/2018)

extracts shows poor correlation (Fig. 4A and B) . However, there was strong correlation in the foldchanges of r-proteins between the experimental replicates (Fig. 5A and B $)$. The lack of correlation between the fold changes in cell extracts and purified ribosomes suggest that a fraction of r-proteins in the cell are not incorporated into ribosomal complexes. We reasoned that either these r-proteins have not yet been successfully incorporated into ribosomal complexes during ribosome biogenesis or ribosomes were disassembling.

To identify differentially abundant r-proteins in purified yeast ribosomes grown in glucose and glycerol, we used a $t$-test of independence with an alpha level of 0.05 . Since there are only minimal sequence differences between the r-protein paralogs to reliably quantify the paralog-specific changes, we reanalyzed the mass spectrometry data using only the unique peptides for quantitation. We quantitated 45 r-proteins with at least one unique peptide (Fig. 6A), and identified 11 r-proteins that were differentially present in the purified ribosome complexes from yeast cells grown in glucose or glycerol (Fig. 6B $)$. The list of significantly changing r-proteins in the purified ribosomes contained the paralog pair RPL8A (eL8A) and RPL8B (eL8B) (97\% protein sequence identity) whose fold changes were opposite to each other. To validate the iTRAQ data, the relative difference in abundance of RPL8A (eL8A) and RPL8B (eL8B) proteins in the purified ribosomes was measured using multiple reaction-monitoring mass spectrometry (Fig. 6C). The data suggest yeast cells have a differential requirement for RPL8A and RPL8B under the two growth conditions.

The cryo-EM data showed $\sim 83 \%$ to $\sim 66 \%$ of the 80 S ribosomes from the purified ribosomal complex missing r-proteins RPS1A/B(eS1) and RPL10(uL16). MS-proteomics successfully identified both rproteins ( $\underline{\text { Table S5 }}$ ). However, the quantitative proteomic analysis showed both proteins increasing their relative abundance in the purified ribosomes $24 \mathrm{~h}$ after changing from glucose to glycerol media, although the change in RPL10 abundance was not statistically significant (Fig 6A-B). To explain the discrepancy, we suspect the sucrose gradient-purified $80 \mathrm{~S}$ ribosomes contained both $80 \mathrm{~S}$ ribosomes as well as sub-80S 
Sun et al.

Identification of changing ribosomes using cryo-EM and MS

EMBL ver $2.8(02 / 12 / 2018)$

particles including 40S, 60S, and partially assembled cytoplasmic ribosomal complexes. Our cryo-EM computational image analysis was able to exclude ribosomal complexes smaller than $80 \mathrm{~S}$ particle while our MS-proteomics analysis could not. We cannot exclude the possibility that Rps1A/B and RPL10 become post-translationally modified when changing growth from glucose to glycerol, thereby distorting our quantitative proteomics analysis. Although the $80 \mathrm{~S}$ ribosomal samples used in the cryo-EM and proteomics experiments were the same, the two analytical methods and resulting computational analysis likely targeted different classes of ribosomal complexes, limiting our ability to directly compare the cryoEM and quantitative proteomic data.

\section{Change of polysomal fractions in response to switch of carbon source}

Since we found that with the shift to glycerol, the percentage of complete $80 \mathrm{~S}$ ribosomes decreased and their r-protein composition changed, we speculated that global translational activities might be affected. We performed polysome profiling experiments using the same time course. These experiments indeed revealed a re-distribution of polysomes (actively translating) into the $80 \mathrm{~S}$ peak (inactive) after 30 min following the shift from glucose to glycerol (Fig. 7). After $450 \mathrm{~min}$, the polysomes partially recovered (Fig. 7). A similar re-distribution of polysomal fractions to the $80 \mathrm{~S}$ fraction was also observed after cells were shifted from glucose to glycerol for $10 \min$ (Kuhn et al., 2001).

The cryo-EM data showed $\approx 20-40 \%$ of the yeast 80 S ribosomes rapidly lose RPL10 and RPS1A/B subunits within $30 \mathrm{~min}$ after the change from glucose to glycerol. Because ribosome biogenesis in eukaryotes is a considerably longer process involving multiple steps and interactions, it is highly unlikely that new ribosomes are being formed within $30 \mathrm{~min}$. We reasoned the cell's $80 \mathrm{~S}$ ribosomes are losing rprotein subunits and adopting an inactive state when shifted from glucose to glycerol. Since the cryo-EM classification method employed is unable to differentiate between 80S ribosomes containing RPL8A (eL8A) and RPL8B (eL8B), we do not know how the subpopulation of ribosomes lacking RPL10 (uL16) and RPS1A/B (eS1) is related to the one exhibiting the change from one r-protein paralog to the other. 
Sun et al.

Identification of changing ribosomes using cryo-EM and MS

EMBL ver $2.8(02 / 12 / 2018)$

RPL8A and RPL8B. Future experiments are needed to confirm whether existing 80S ribosomes are exchanging RPL8B with cytoplasmic RPL8A or new ribosomal synthesis is required to change these $\mathrm{r}$ protein paralogues in the cell's population of ribosomal subunits.

\section{Different functional Roles of RPL8A and RPL8B}

Using growth assays, we tested the paralog-specific roles of $R P L 8 A$ and $R P L 8 B$ using yeast genetics. When we compared yeast growth rates in glucose versus glycerol, we observed a functional difference in the activity or specificity of the yeast ribosome depending on the presence of eL8A and eL8B (Fig. 8). Under optimal growth conditions in rich media with glucose as the carbon source, the function of either rpl8a $\Delta$ or $r p l 8 b \Delta$ null allele can be compensated for by the paralogous gene (Fig. 8A). However, when the cells encounter non-optimal growth in glycerol, the differential requirement for each paralog becomes apparent (Fig. 8B-D). This result is consistent with the original ribosome filter hypothesis in which the ribosome filter was proposed to fine-tune gene expression (Mauro and Edelman, 2002; Mauro and Edelman, 2007).

\section{Discussion}

In this study we have employed both cryo-EM experiments and quantitative mass spectrometry along with polysome profiling and genetic analysis to follow changes in the yeast ribosome composition as a function of time after a shift from growth in glucose to glycerol media. Using a novel quantitative approach combining pooled classification with 3D visualization, our cryo-EM experiments identified and tracked subpopulations of ribosomes lacking individual r-proteins. The cryo-EM experiments enabled us to identify changes in $80 \mathrm{~S}$ structure, protein composition, and bound tRNAs after switching from growth in glucose to glycerol. Using classification tools, we could pre-process the cryo-EM images to select only $80 \mathrm{~S}$ particles for in-depth analysis and exclude smaller ribosomal particles. The quantitative proteomic experiments enabled us to identify and quantify changing r-proteins, including paralogs, incorporated into yeast ribosome populations that would be impossible to identify with the current cryo- 
Sun et al.

Identification of changing ribosomes using cryo-EM and MS

EMBL ver 2.8 (02/12/2018)

EM methodology. Isotopic labeling enabled us to perform precise quantitative measurements of individual r-proteins in the purified ribosomal complexes. Unlike cryo-EM's 80S/60S/40S classification capability, the quantitative proteomic experiments analyzed mixtures of purified ribosomal complexes isolated from sucrose gradients. The r-proteins' low molecular weight and high abundance of lysine and arginine residues limited the number of tryptic peptides that could be surveyed by our mass spectrometry approach. Therefore, the number of r-proteins that could be confidently identified and quantified by the quantitative mass spectrometry was limited. Targeted mass spectrometry approaches would be one solution to this limitation. Overall, combining the two approaches of cryo-EM and mass spectroscopy allowed us to identify changing $80 \mathrm{~S}$ ribosome compositions, structure, and functional status that would escape detection if only a single approach was employed.

Our identification of ribosomes with sub-stoichiometric compositions of r-proteins presents intriguing possibilities. The data analysis resulting in reconstructions of selected classes uncovered a sizeable fraction of translation-incompetent yeast ribosomes lacking both RPS1A/B (eS1) and RPL10 (uL16) in cells growing in glucose. Remarkably, this fraction almost doubled immediately (within 30 min) after the shift from glucose to glycerol. Apparently, an increasing number of the r-proteins RPL10 (uL16) and/or RPS1A/B (eS1) dissociate from existing complete ribosomes when yeast cells are grown in glycerol. Depletion of the two r-proteins from the ribosome could be functioning as a posttranscriptional regulatory mechanism to slow the overall rate of protein synthesis as both genes are essential in yeast (Arevalo and Warner, 1990). It is important to note that the presence of both proteins in the ribosome is essential for translation. RPS1A/B (eS1) forms part of the protein-protein and r-protein-rRNA contacts between two adjacent 40S subunits in polysomes (Myasnikov et al., 2014), and RPL10 (uL16) plays a central role in the interaction of the ribosome with GTP-bound translation factors. The rapid loss of RPL10 (uL16) and/or RPS1A/B (eS1) from the 80S ribosome within $30 \mathrm{~min}$ after the change from glucose to glycerol suggests that the environmental change induces the loss of these two r-proteins from the existing pool of 
Sun et al.

Identification of changing ribosomes using cryo-EM and MS

EMBL ver 2.8 (02/12/2018)

complete ribosomes, rather than the production of specialized ribosomes lacking these two proteins during the process of ribosomal biogenesis (Gasch et al., 2000). While environmental changes, such as the switch from glucose to glycerol, induce rapid changes in yeast transcriptional profiles (Gasch et al., 2000), ribosome biogenesis is a relatively slow process requiring multiple steps and factors (Woolford and Jonathan, 1991). Surprisingly, the proportion of complete, translation-competent ribosomes does not recover to the prior state. Since yeast cells grow slowly with glycerol as a carbon source compared to glucose, the ribosomes lacking both of the r-proteins may be part of a non-translating reserve ribosome pool (Samir et al., 2015).

\section{Author Contributions}

A.J.L. conceived the hypothesis. M.S., P.S., B.S., J.F., and A.J.L. designed the experiments. M.S. and B.S. performed cryo-EM experiments. M.S., B.S., and W. L. analyzed the cryo-EM results. P.S. and C.M.B. performed the iTRAQ, liquid chromatograph mass spectrometry and polysome profiling experiments. P.S. and Rahul analyzed the MS results. M.S., P.S., J.F. and A.J.L. wrote the manuscript. All authors approved the final manuscript.

\section{Acknowledgements}

This study was supported by HHMI and NIH R01 GM29169 (to J.F.) and NIH RO1 grant GM64779 (to AL). CB was supported by NIH training grant T32 AI007611. 
Sun et al.

Identification of changing ribosomes using cryo-EM and MS

EMBL ver $2.8(02 / 12 / 2018)$

\section{Figure Legends}

Fig. 1. The $\log _{2}$ fold-changes in the distributions of $40 \mathrm{~S}$ and $60 \mathrm{~S}$ subunit protein abundances caused by changes in growth conditions. Quantitative proteomics was used to identify and quantify changes in individual r-proteins abundances from S. cerevisiae whole-cell protein extracts after shifts in carbon source and temperature (Samir et al., 2015). Yeast was grown at standard conditions (glucose, $30^{\circ} \mathrm{C}$ ) and changed to three different environmental growth conditions or stimuli: high temperature $39^{\circ} \mathrm{C}$ (HT, blue); glycerol (G, green), and high temperature and glycerol combined (HT+G, red). A custom Python script CompZilla.py was used to analyze changes in the r-proteins in the quantitative proteomic data. Open circles represent $r$-protein outliers in the $\log _{2}$ fold-changes in their protein abundance.

Fig. 2. Cryo-EM reconstructions of $S$. cerevisiae $80 \mathrm{~S}$ ribosomes after the switch from glucose to glycerol. Yellow, 40S subunit; blue, 60S subunit. In all panels. A-E, two different views of the ribosome are shown, related by a $\sim 90$-degree rotation around a vertical axis in the plane of the figure. On the right hand side in each panel, a magnified slab of a region marked in the left view is shown, with densities shown in transparency. (A) Density map reconstructed from the sub-population of incomplete 80S ribosomes that lack only the density for RPL10 (uL16) protein but possess protein RPS1A/B (eS1) (orange). (B) Rotated incomplete $80 \mathrm{~S}$ ribosomes that lack the density for both RPS1A/B (eS1) and RPL10 (uL16) r-proteins, as well as the interacting partners of RPS1A/B (eS1),RPS26A/B (eS26), and RPS14A/B (uS11). (C) Non-rotated incomplete 80S ribosomes lacking the densities for both RPS1A/B (eS1) and RPL10 (uL16) r-proteins. (D, E) Incomplete 80S ribosomes lacking densities for both the RPS1A/B (eS1) and RPL10 (uL16) proteins, as well as the H38 of the $28 \mathrm{~S}$ rRNA. The interacting partners of RPS1A/B (eS1) (RPS26A/B (eS26) and RPS14A/B (uS11)) are also missing entirely. The xray structures of S. cerevisiae (PDB 4V88) 40S and 60S subunits were rigid-body fitted separately into cryo-EM densities using UCSF Chimera (Pettersen et al., 2004). 
Sun et al.

Identification of changing ribosomes using cryo-EM and MS

EMBL ver $2.8(02 / 12 / 2018)$

Fig. 3. Structural inventory as a function of time. The $x$-axis represents the time (min, $\log$ scale) that cells were incubated in glycerol medium. The y-axis represents the percentage of complete 80S ribosomes. Generally, the percentage of complete $80 \mathrm{~S}$ ribosomes in each sample set decreased upon onset of the glucose-to-glycerol switch. This change reflects the general decrease in binding of RPS1A/B (eS1) and RPL10 (uL16) to 80S ribosomes.

Fig. 4. Correlation analysis between the fold changes of r-proteins in whole cell extracts and purified ribosomes from cells grown at standard conditions (glucose, $30^{\circ} \mathrm{C}$ ) and glycerol (glycerol, $3^{\circ} \mathbf{C}$ ). (See Tables S2 and S3). The summary of three replicates is shown. (A) Cross-correlation matrix. Numbers represent Pearson's R. W\# represent whole extract replicates. R\# represent purified ribosome replicates. (B) Scatter plot showing the relationship between fold-changes in whole cell extracts (x-axis) and purified ribosomes (y-axis).

Fig. 5. Scatterplots showing reproducibility among replicates of whole cell extracts and purified ribosomes. (A) Whole-cell extract replicates. (B) Purified ribosome replicates. Plots were generated in RStudio.

Fig. 6. Quantitation of r-proteins in ribosomes purified from yeast cells grown in glucose and glycerol. (A) Forty-five ribosomal proteins identified and quantitated using at least one unique peptide in iTRAQ-mass spectrometry experiments. The $\log _{2}$ transformed fold change in the r-protein's relative abundance $24 \mathrm{~h}$ after changing growth in glucose to glycerol was used to generate the heatmap. Eleven statistically significant differentially abundant r-proteins in the purified ribosomes from cells grown in glucose and glycerol identified using a t-test's $p$-value less than 0.05 . (C) The protein abundance of RPL8A and RPL8B validated using multiple reaction monitoring in three independently purified ribosome replicates. Color Legend: red increased; green decreased abundance of the yeast r-protein in cells growing in glycerol compared to glucose media. 
Sun et al.

Identification of changing ribosomes using cryo-EM and MS

EMBL ver $2.8(02 / 12 / 2018)$

Fig. 7. The switch of carbon source from glucose to glycerol inhibits translation. Polyribosome traces

from the wild-type S. cerevisiae strain. Yeast was grown in complete medium containing glucose, then resuspended at $\mathrm{T}=0$ in medium containing glycerol for the indicated times (min). Polyribosomes were analyzed as described in 'STAR Methods'. The peaks that contain the small 40S ribosomal subunit, the large $60 \mathrm{~S}$ ribosomal subunit, and complete $80 \mathrm{~S}$ ribosomes are indicated by arrows. The polysome peaks are bracketed.

Fig. 8. Doubling times comparisons. A \& B) Stars denote the statistical significance of comparison with the WT strain $(p$-values $<0.05)$. A) Doubling times of WT, rpl8a, and $r p l 8 b$ with glucose as carbon source. B) Doubling times of WT, $r p l 8 a$, and $r p l 8 b$ with glycerol as carbon source. C) Doubling times of $r p l 8 a$ with Rpl8a on plasmid, $r p l 8 a$ with Rpl8b on plasmid, $r p l 8 b$ with Rpl8b on plasmid, and $r p l 8 b$ with Rpl8a on plasmid with glucose as carbon source. D) Doubling times of rpl8a with Rpl8a on plasmid, rpl8a with Rpl8b on plasmid, rpl8b with Rpl8b on plasmid, and rpl8b with Rpl8a on plasmid with glycerol as carbon source. Stars denote the statistical significance of comparison denoted by the lines above the bar graph ( $p$-values $<0.05)$.

Figure S1. Full framework of 'pooled' classification strategy and results (Related to Figure 5). (A) Schematic diagram of our 'pooled classification' data processing procedure. Samples purified from six time points were pooled together and classified using the same procedure. Further analysis was based mainly on quantitative jumper analysis (20) with minimum user interference. (B) Summary of the fullscale cryo-EM studies. "ㄱ", present; "X”, absent. Cryo-EM reconstructions of incomplete 80S ribosomes are presented in front view and colored in light blue (B, left), while complete $80 \mathrm{~S}$ ribosomes are colored in grey (B, right). Resolutions reported are based throughout on the 'gold standard' protocol along with the FSC $=0.143$ criterion, and involved soft masking and high-resolution noise substitution (Chen et al., 2013). 
bioRxiv preprint doi: https://doi.org/10.1101/271833; this version posted February 26,2018 . The copyright holder for this preprint (which was not certified by peer review) is the author/funder, who has granted bioRxiv a license to display the preprint in perpetuity. It is made available under aCC-BY-NC-ND 4.0 International license.

Sun et al.

Identification of changing ribosomes using cryo-EM and MS

EMBL ver $2.8(02 / 12 / 2018)$

Figure S2. Global conformational changes of incomplete 80 S ribosomes (Related to Fig 2). (A) Intersubunit rotation and 40S subunit head-swiveling movements in the incomplete 80S (class 1). Comparison of the 40S subunit positions in nrt80S-P-E state (yellow) and the incomplete $80 \mathrm{~S}$ (class 1) (orange). (B) Head-swiveling movements in the incomplete $80 \mathrm{~S}$ (class 3). The position of eS1 on nrt80S-P-E state is highlighted by a red circle. Comparisons were obtained by structural alignment on the 60S subunits of the 80S ribosomes using UCSF Chimera (Pettersen et al., 2004).

\section{Methods}

Data and Software Availability

\begin{tabular}{|c|c|c|}
\hline \multicolumn{3}{|c|}{ Software and Algorithms } \\
\hline Leginon & Suloway et al., 2005 & $\begin{array}{l}\text { http://emg.nysbc.org/redmine/projects/leginon/ } \\
\text { wiki/Leginon_Homepage }\end{array}$ \\
\hline MotionCorr & Li et al., 2013 & http://cryoem.ucsf.edu/software/driftcorr.html \\
\hline Arachid & Langlois et al., 2014 & http://www.arachnid.us/ \\
\hline RELION v1.2 & Scheres, 2012 & http://www2.mrc-lmb.cam.ac.uk/relion \\
\hline UCSF Chimera & Pettersen et al., 2004 & http://www.cgl.ucsf.edu/chimera/ \\
\hline$R$ Studio & RStudio & https://www.rstudio.com/ \\
\hline $\begin{array}{c}\text { Proteome } \\
\text { Discoverer } 1.4\end{array}$ & Thermo Scientific & $\begin{array}{l}\text { https://www.thermofisher.com/order/catalog/ } \\
\text { product/IQLAAEGABSFAKJMAUH }\end{array}$ \\
\hline
\end{tabular}

\section{Contact for Reagent and Resource Sharing}

Requests for reagents may be directed to the lead contacts Andrew J. Link (andrew.link@vanderbilt.edu ) and Joachim Frank (jf2192@cumc.columbia.edu) 
Sun et al.

Identification of changing ribosomes using cryo-EM and MS

EMBL ver $2.8(02 / 12 / 2018)$

\section{Experimental Model and Subject Details}

\section{Strains and Media}

All experiments used the diploid S. cerevisiae strain BY4743, which has been previously described (Brachmann et al., 1998). Cells were grown using standard techniques (Amberg, 2005). The genomic region of RPL8a and RPL8b, including $\sim 1 \mathrm{~kb}$ upstream and $\sim 200 \mathrm{~b}$ downstream, were cloned into the pFA6a-His3MX6 plasmid with Gibson cloning (New England Biolabs) to generate plasmids pALL8a and pALL8b, respectively (Gibson et al., 2009). Strains were transformed using the standard yeast transformation protocol (Amberg et al., 2005).

For calculating doubling times in YPD and YPG media, $5 \mathrm{~mL}$ starter cultures from a single

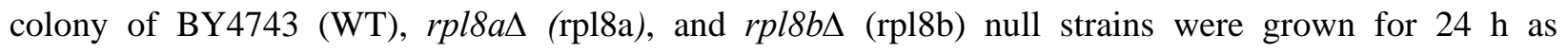
previously described (Samir et al., 2015). For calculating doubling times of $r p l 8 a \Delta$ and $r p l 8 b \Delta$ strains complemented with plasmids pALL8a or pALL8b, yeast was grown in SC-His glucose (ScD) or SC-His glycerol (ScG) as previously described (Samir et al., 2015; Baker Brachmann et al., 1998; Winzeler et al., 1999).

For plasmid rescue experiments, yeast strains were grown in SC-His supplemented with G418. One hundred microliters of the different media were added to wells in a 96-well plate as shown in Table S4. Cells were grown at $30^{\circ} \mathrm{C}$ in a Synergy Biotek plate reader for 720 min with constant shaking. OD 660 readings were taken every $3 \mathrm{~min}$. A text file containing the OD 660 readings was exported and parsed using a custom Python script. Doubling times were calculated using the $l m$ function in $R$. A Student's $t$ test of independence was used to calculate the statistical significances at alpha level of 0.05. All $p$-values were adjusted for multiple hypotheses testing using the Bonferroni correction (Dunn, 1959, 1961). The source code used in this project is found in supplementary data. 
Sun et al.

Identification of changing ribosomes using cryo-EM and MS

EMBL ver $2.8(02 / 12 / 2018)$

\section{Method Details}

\section{Ribosome isolation and purification}

S. cerevisiae cells were grown in YPD medium (1\% yeast extract, $2 \%$ peptone, $2 \%$ glucose) and shifted to YPG (1\% yeast extract, $2 \%$ peptone, 3\% glycerol). At the following time points ribosomes were isolated and purified: $0 \mathrm{~min}, 30 \mathrm{~min}, 120 \mathrm{~min}, 240 \mathrm{~min}, 450 \mathrm{~min}$ and $1440 \mathrm{~min}$. Cells were first centrifuged at $2000 \mathrm{rpm}$ for $5 \mathrm{~min}$ at $4^{\circ} \mathrm{C}$ using a Sorvall HLR6/H600A/HBB6 rotor in a Sorvall RC-3B centrifuge and washed with ice cold deionized $\mathrm{H}_{2} \mathrm{O}$. The pellets were resuspended in $1 \mathrm{~mL}$ ice cold wash buffer (10 mM Tris pH 8, $5 \mathrm{mM} \beta$-mercaptoethanol, $500 \mathrm{mM}$ ammonium chloride, $100 \mathrm{mM}$ magnesium acetate) and lysed at $4^{\circ} \mathrm{C}$ for 10 min using glass beads and a Bead Beater (BioSpec, Inc) as previously described (Browne et al., 2013). The cell suspensions were clarified by centrifugation at 20,000g for 15 $\min$ at $4{ }^{\circ} \mathrm{C}$. The pellets were discarded and the supernatants were overlaid onto 5-20\% discontinuous sucrose gradients prepared in wash buffer. The gradients were centrifuged at 28,000 rpm using a SW-41 swinging bucket rotor for $18 \mathrm{~h}$ at $4^{\circ} \mathrm{C}$. The ribosome-enriched pellets were resuspended in $1 \mathrm{~mL}$ ice cold standard buffer $(10 \mathrm{mM}$ Tris $\mathrm{pH} 8,5 \mathrm{mM} \beta$-mercaptoethanol, $50 \mathrm{mM}$ ammonium chloride, $5 \mathrm{mM}$ magnesium acetate) and centrifuged for $10 \mathrm{~min}$ at $10,000 \mathrm{~g}$ at $4^{\circ} \mathrm{C}$. The pellets were discarded, and the ribosome suspensions were stored at $-80^{\circ} \mathrm{C}$.

\section{iTRAQ labeling, liquid chromatography mass spectrometry, and data analysis}

iTRAQ labeling, liquid chromatography tandem mass spectrometry, and database searches for peptide spectrum matching were done as previously described (Eng et al., 1994; Link et al., 1999; Samir et al., 2015). Proteome Discoverer 1.4 (Thermo Scientific) output files were imported into ProteoIQ (Premier BioSoft) for protein assembly and reporter ion quantitation. Peptide and protein FDR was set to 5\%. Reporter ion normalization was based upon their intensities for the single copy r-proteins. This was 
Sun et al.

Identification of changing ribosomes using cryo-EM and MS

EMBL ver $2.8(02 / 12 / 2018)$

done for both purified ribosome experiments and the previously published whole cell extract experiment (Samir et al., 2015). The purified ribosome experiment was analyzed in two ways. For comparison with the whole cell extract analysis, all peptides were used for quantitation and protein assembly. To identify differentially present r-proteins, only the peptides uniquely mapping to one r-protein were used for quantitation and protein assembly.

Proteotypic peptides were selected for targeted quantitation from a database of identified peptides in the iTRAQ experiments. Transitions for unscheduled scout experiments were selected based upon NIST and GPM spectral libraries. Fifty $\mu \mathrm{g}$ of the purified ribosomes was digested with sequencing grade modified trypsin (1:50; Promega Corporation) and desalted essentially as described (Samir et al., 2015). Peptides were eluted using an elution buffer composition of $50 \%$ acetonitrile, $0.1 \%$ trifluoroacetic acid. Peptides were analyzed using a 90 min scheduled SRM analysis. Briefly, peptides were autosampled onto a $200 \mathrm{~mm}$ by $0.1 \mathrm{~mm}$ (Jupiter 3 micron, 300A), analytical column coupled directly to an TSQVantage (ThermoFisher) using a nanoelectrospray source and resolved using an aqueous to organic gradient (1-45\% Buffer B) at a $500 \mathrm{nl} / \mathrm{min}$ flow rate. Using a series of unscheduled scout runs to determine retention times and transitions to monitor, a scheduled instrument method encompassing a 10 min window around each retention time along with calculated collision energies was created using Skyline (MacLean et al., 2010). Q1 peak width resolution was set to 0.7, collision gas pressure was 1 mTorr, and the EZmethod cycle time was $5 \mathrm{~s}$.

The resulting RAW instrument files were imported into Skyline for peak-picking and quantitation (MacLean et al., 2010). The peak areas of the transitions were exported, and further analysis was done in Microsoft Excel. The sum of the peak areas of all the transitions of a given peptide, the peptide peak area, was used as the quantitative measure of abundance for the peptide. The average of peptide peak areas of all the peptides from a given protein, the protein peak area, was used as the quantitative measure of abundance of the protein. The average protein peak areas of the single copy r-protein RPL5p was used as 
Sun et al.

Identification of changing ribosomes using cryo-EM and MS

EMBL ver $2.8(02 / 12 / 2018)$

a control. For differential analysis, in the first step, a ratio of peak area of the test protein to the peak area of the control was calculated across all samples. In the next step, a two sample $t$-test with alpha level 0.05 was performed with the ratios to test for statistical significance. Finally, the fold change was calculated by taking the ratio of the average of ratios. The calculated fold change was $\log 2$ transformed.

\section{COMPzilla}

COMPzilla uses CYC2008 2.0, a manually curated database of biomolecular complexes in yeast to identify complexes that are differentially present (Pu et al., 2007). In the first step COMPzilla creates a dictionary with complex names as keys and the proteins that constitute the complex as values. Next, COMPzilla creates a dictionary in which complex names are still the keys, but values are mapped to fold changes of the proteins that constitute the complex. In the third step, COMPzilla creates a list all the fold changes in the population for statistical testing. Finally, COMPzilla compares the fold change distributions associated with protein complexes with population fold change distribution using a two sample $t$-test of independence and two-sample Kolomogorv-Smirnov test. COMPzilla exports the results of the two tests in separate tab delimited text files, in which the first column contains the complex names, the second column contains t-statistics or ks-statistics, and the third column contains the corresponding $p$ value. The CompZilla.py source code is available in the supplementary data.

\section{Polysome profiling}

Polysome profiling was modified from previous methods (Browne et al., 2013). S. cerevisiae cells were grown in YPD to mid-log phase. Strains were then transferred to YPG and allowed to continue to grow. At $30 \mathrm{~min}, 120 \mathrm{~min}, 240 \mathrm{~min}$ and $450 \mathrm{~min}$, cells were collected and lysed as previously described. Protein extracts were layered on top of $7 \%-47 \%$ sucrose gradients and centrifuged. 
Sun et al.

Identification of changing ribosomes using cryo-EM and MS

EMBL ver $2.8(02 / 12 / 2018)$

\section{Cryo-electron microscopy}

Four microliters of purified ribosomes was applied to holey carbon grids (carbon-coated Quantifoil R2/4 grid, Quantifoil Micro Tools, GmbH, Großlöbichau, Germany) containing an additional continuous thin layer of carbon, and glow-discharged using Gatan Solarus 950 (Grassucci et al., 2007). Grids were blotted for $4 \mathrm{~s}$ at $4^{\circ} \mathrm{C}$ in $100 \%$ humidity and vitrified by plunging into liquid ethane cooled with liquid nitrogen, using the Mark IV Vitrobot (FEI, Hillsboro, Oregon) (Dubochet et al., 1988).

For the initial studies, five data sets $(\mathrm{T}=0,30,120,240,450$, and $1440 \mathrm{~min})$ were collected on an FEI Tecnai F20 electron microscope (FEI, Hillsboro, Oregon) operating at $200 \mathrm{kV}$, equipped with a $4 \mathrm{k}$ x 4k CCD camera (Gatan, Pleasanton, CA). Images were recorded using the automated data collection system Leginon (Suloway et al., 2005), and taken at the magnification of 5,000 x, corresponding to a calibrated pixel size of $2.245 \AA$.

For the full-scale study, data $(\mathrm{T}=0,30,120,240,450$ and $1440 \mathrm{~min})$ were collected on a TF30 Polara electron microscope (FEI, Hillsboro, Oregon) operating at $300 \mathrm{kV}$, set up with a K2 Summit direct electron detection camera (Gatan, Warrendale, PA). Images were recorded using the automated data collection system, Leginon (Suloway et al., 2005), in counting mode, and taken at the nominal magnification of $23,000 \mathrm{x}$, corresponding to a calibrated pixel size of $1.66 \AA$. The dose rate was nominally set to 8 electron counts per physical pixel per second (Liao et al., 2013), and the total exposure time was 8 seconds. Image stacks were collected in a defocus range of $-1.5 \mu \mathrm{m}$ to $-3.5 \mu \mathrm{m}$ and fractionated into 20 frames, each with an exposure time of $0.4 \mathrm{~s}$.

\section{Image processing}

The six time-resolved sample sets were pooled and processed together. First, the dosefractionated image stacks were corrected for beam-induced motion, using the method of Li et al. (Li et al., 2013), and averages of all 20 frames were used for image processing. A total number of $\sim 291,000$ 
Sun et al.

Identification of changing ribosomes using cryo-EM and MS

EMBL ver 2.8 (02/12/2018)

particles were analyzed from 6576 selected frame-averaged micrographs. Particles were picked using the ara-autopick and ara-crop tools in Arachnid (Langlois et al., 2014), and the contrast transfer function parameters were estimated using the sp-defocus tool in Arachnid (Langlois et al., 2014). 3D classification was performed using RELION software (version 1.2) (Scheres, 2012) to discard defective particles and identify structurally homogeneous subsets.

Initial RELION 3D classification, with $\mathrm{K}=10$ classes and an angular sampling of $1.8^{\circ}$, revealed two major populations, namely $80 \mathrm{~S}$ ribosomes that are complete, and $80 \mathrm{~S}$ ribosomes that lack densities for uL16 r-protein and/or eS1 r-protein, (denoted as 'complete 80S' and 'incomplete 80S', respectively). Since reconstructions from both populations showed fragmented densities, we performed a second tier of exhaustive 3D classification to further explore the heterogeneity. We quantitatively and qualitatively analyzed the classification results by (i) quantitative jumper analysis (Chen et al., 2014) and (ii) prior structural knowledge of $S$. cerevisiae $80 \mathrm{~S}$ ribosomes. In this way, we re-grouped classes which are conformationally and compositionally similar, and performed RELION auto-refinement on each class. (Figure S1).

In total, we found 10 major subpopulations, 5 complete $80 \mathrm{~S}$ populations and 5 incomplete $80 \mathrm{~S}$ populations. For the population of complete $80 \mathrm{~S}$ ribosomes, we have (i) rotated $80 \mathrm{~S}$ bound with P/E tRNA, with an average resolution of $\sim 5.6 \AA$, (ii) rotated $80 \mathrm{~S}$ with P/E tRNA, $\sim 6.3 \AA$, (iii) rotated $80 \mathrm{~S}$ with

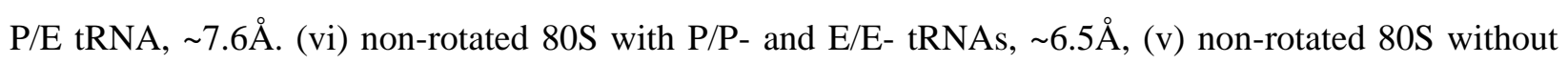
any tRNAs, $\sim 6.4 \AA$. The first three rotated 80 S classes are different from each other by the degree of intersubunit rotation and head movements, in comparison with class 4, nrt80S-P/E (Figure S1 and Table S3).

For the subpopulations of incomplete $80 \mathrm{~S}$ ribosomes, we found (i) rotated $80 \mathrm{~S}$, lacking only the density for uL16, with an average resolution of $\sim 9.4 \AA$, (ii) rotated $80 \mathrm{~S}$, lacking densities for both uL16 and eS1, $6.1 \AA$, (iii) non-rotated $80 \mathrm{~S}$ lacking densities for both uL16 and eS1, $6.5 \AA$, (vi) non-rotated 80S lacking densities for both uL16 and eS1, as well as densities for small fragments of rRNA, 9.2 Å, (v) 
Sun et al.

Identification of changing ribosomes using cryo-EM and MS

EMBL ver 2.8 (02/12/2018)

non-rotated 80S lacking densities for both $\mathrm{uL} 16$ and $\mathrm{eS} 1$, as well as densities for small fragments of rRNA, 9.8 (Fig. 5, Fig. S1 and Table S4).

Resolutions reported throughout were based on the 'gold standard' protocol along with the FSC = 0.143 criterion, and involved soft masking and high-resolution noise substitution (Chen et al., 2013; Scheres, 2012). For the final visualization, all density maps were corrected for the effects of a soft mask in RELION post-processing (Chen et al., 2013; Scheres, 2012), and sharpened by application of an automatically estimated negative b-factor (Scheres, 2012).

\section{References}

Amberg, D.C. (2005). Methods in yeast genetics : a Cold Spring Harbor Laboratory course manual, D. Burke, J.N. Strathern, and D. Burke, eds. (Cold Spring Harbor, N.Y. :: Cold Spring Harbor Laboratory Press).

Amberg, D.C., Burke, D.J., and Strathern, J.N. (2005). Methods in Yeast Genetics: A Cold Spring Harbor Laboratory Course Manual, 2005 Edition, 2005 Edition edn (Long Island, New York.: Cold Spring Harbor Laboratory Press).

Arevalo, S.G., and Warner, J.R. (1990). Ribosomal protein L4 of Saccharomyces cerevisiae: the gene and its protein. Nucleic Acids Res 18, 1447-1449.

Baker Brachmann, C., Davies, A., Cost, G.J., Caputo, E., Li, J., Hieter, P., and Boeke, J.D. (1998). Designer deletion strains derived from Saccharomyces cerevisiae S288C: A useful set of strains and plasmids for PCR-mediated gene disruption and other applications. Yeast 14, 115-132.

Bauer, J.W., Brandl, C., Haubenreisser, O., Wimmer, B., Weber, M., Karl, T., Klausegger, A., Breitenbach, M., Hintner, H., von der Haar, T., et al. (2013). Specialized yeast ribosomes: a customized tool for selective mRNA translation. PLoS One 8, e67609. 
Sun et al.

Identification of changing ribosomes using cryo-EM and MS

EMBL ver 2.8 (02/12/2018)

Brachmann, C.B., Davies, A., Cost, G.J., Caputo, E., Li, J., Hieter, P., and Boeke, J.D. (1998). Designer deletion strains derived from Saccharomyces cerevisiae S288C: a useful set of strains and plasmids for PCR-mediated gene disruption and other applications. Yeast 14, 115-132.

Breslow, D.K., Cameron, D.M., Collins, S.R., Schuldiner, M., Stewart-Ornstein, J., Newman, H.W., Braun, S., Madhani, H.D., Krogan, N.J., and Weissman, J.S. (2008). A comprehensive strategy enabling high-resolution functional analysis of the yeast genome. Nat Methods 5, 711-718.

Browne, C.M., Samir, P., Fites, J.S., Villarreal, S.A., and Link, A.J. (2013). The yeast eukaryotic translation initiation factor $2 \mathrm{~B}$ translation initiation complex interacts with the fatty acid synthesis enzyme YBR159W and endoplasmic reticulum membranes. Mol Cell Biol 33, 1041-1056.

Carpenter, S., Ricci, E.P., Mercier, B.C., Moore, M.J., and Fitzgerald, K.A. (2014). Post-transcriptional regulation of gene expression in innate immunity. Nat Rev Immunol 14, 361-376.

Chen, B., Shen, B., and Frank, J. (2014). Particle migration analysis in iterative classification of cryo-EM single-particle data. J Struct Biol 188, 267-273.

Chen, S., McMullan, G., Faruqi, A.R., Murshudov, G.N., Short, J.M., Scheres, S.H., and Henderson, R. (2013). High-resolution noise substitution to measure overfitting and validate resolution in 3D structure determination by single particle electron cryomicroscopy. Ultramicroscopy $135,24-35$.

Costa-Mattioli, M., Sossin, W.S., Klann, E., and Sonenberg, N. (2009). Translational Control of LongLasting Synaptic Plasticity and Memory. Neuron 61, 10-26.

Dever, T.E., and Green, R. (2012). The Elongation, Termination, and Recycling Phases of Translation in Eukaryotes. Cold Spring Harbor Perspectives in Biology 4.

Dubochet, J., Adrian, M., Chang, J.J., Homo, J.C., Lepault, J., McDowall, A.W., and Schultz, P. (1988). Cryo-electron microscopy of vitrified specimens. Q Rev Biophys 21, 129-228.

Dunn, O.J. (1959). Estimation of the Medians for Dependent Variables. 192-197. 
Sun et al.

Identification of changing ribosomes using cryo-EM and MS

EMBL ver 2.8 (02/12/2018)

Dunn, O.J. (1961). Multiple Comparisons Among Means. Journal of the American Statistical Association $56,52-64$.

Eng, J.K., McCormack, A.L., and Yates, J.R. (1994). An Approach to Correlate Tandem Mass-Spectral Data of Peptides With Amino-Acid-Sequences in a Protein Database. J Am Soc Mass Spectr 5, 976989.

Frank, J. (2006). Three-Dimensional Electron Microscopy of Macromolecular Assemblies : Visualization of Biological Molecules in Their Native State. Oxford University Press, New York

Frank, J., and Gonzalez, R.L., Jr. (2010). Structure and dynamics of a processive Brownian motor: the translating ribosome. Annu Rev Biochem 79, 381-412.

Gasch, A.P., Spellman, P.T., Kao, C.M., Carmel-Harel, O., Eisen, M.B., Storz, G., Botstein, D., and Brown, P.O. (2000). Genomic expression programs in the response of yeast cells to environmental changes. Mol Biol Cell 11, 4241-4257.

Giaever, G., Chu, A.M., Ni, L., Connelly, C., Riles, L., Veronneau, S., Dow, S., Lucau-Danila, A., Anderson, K., Andre, B., et al. (2002). Functional profiling of the Saccharomyces cerevisiae genome. Nature 418, 387-391.

Gibson, D.G., Young, L., Chuang, R.-Y., Venter, J.C., Hutchison, C.A., and Smith, H.O. (2009). Enzymatic assembly of DNA molecules up to several hundred kilobases. Nat Meth 6, 343-345.

Grassucci, R.A., Taylor, D.J., and Frank, J. (2007). Preparation of macromolecular complexes for cryoelectron microscopy. Nat Protoc 2, 3239-3246.

Hinnebusch, A.G. (2015). Translational control 1995-2015: unveiling molecular underpinnings and roles in human biology. RNA 21, 636-639.

Holcik, M., and Sonenberg, N. (2005). Translational control in stress and apoptosis. Nat Rev Mol Cell Biol 6, 318-327. 
Sun et al.

Identification of changing ribosomes using cryo-EM and MS

EMBL ver 2.8 (02/12/2018)

Woolford, Jr, J. L and Warner, J.R. (1991). The Ribosome and Its Synthesis. Cold Spring Harbor Monograph Archive; Volume 21A (1991): Volume I: The Molecular and Cellular Biology of the Yeast $<\mathrm{em}>$ Saccharomyces $</ \mathrm{em}>$ : Genome Dynamics, Protein Synthesis, and Energetics.

Kapasi, P., Chaudhuri, S., Vyas, K., Baus, D., Komar, A.A., Fox, P.L., Merrick, W.C., and Mazumder, B. (2007). L13a blocks 48S assembly: role of a general initiation factor in mRNA-specific translational control. Mol Cell 25, 113-126.

Kapp, L.D., and Lorsch, J.R. (2004). The Molecular Mechanics of Eukaryotic Translation. Annual Review of Biochemistry 73, 657-704.

Komili, S., Farny, N.G., Roth, F.P., and Silver, P.A. (2007). Functional Specificity among Ribosomal Proteins Regulates Gene Expression. Cell 131, 557-571.

Kondrashov, N., Pusic, A., Stumpf, C.R., Shimizu, K., Hsieh, Andrew C., Xue, S., Ishijima, J., Shiroishi, T., and Barna, M. (2011). Ribosome-Mediated Specificity in Hox mRNA Translation and Vertebrate Tissue Patterning. Cell 145, 383-397.

Kong, J., and Lasko, P. (2012). Translational control in cellular and developmental processes. Nat Rev Genet 13, 383-394.

Kuhn, K.M., DeRisi, J.L., Brown, P.O., and Sarnow, P. (2001). Global and specific translational regulation in the genomic response of Saccharomyces cerevisiae to a rapid transfer from a fermentable to a nonfermentable carbon source. Mol Cell Biol 21, 916-927.

Langlois, R., Pallesen, J., Ash, J.T., Nam Ho, D., Rubinstein, J.L., and Frank, J. (2014). Automated particle picking for low-contrast macromolecules in cryo-electron microscopy. J Struct Biol 186, 1-7.

Lee, A.S., Burdeinick-Kerr, R., and Whelan, S.P. (2013). A ribosome-specialized translation initiation pathway is required for cap-dependent translation of vesicular stomatitis virus mRNAs. Proc Natl Acad Sci U S A 110, 324-329. 
Sun et al.

Identification of changing ribosomes using cryo-EM and MS

EMBL ver 2.8 (02/12/2018)

Li, X., Mooney, P., Zheng, S., Booth, C.R., Braunfeld, M.B., Gubbens, S., Agard, D.A., and Cheng, Y. (2013). Electron counting and beam-induced motion correction enable near-atomic-resolution singleparticle cryo-EM. Nat Methods 10, 584-590.

Liao, M., Cao, E., Julius, D., and Cheng, Y. (2013). Structure of the TRPV1 ion channel determined by electron cryo-microscopy. Nature 504, 107-112.

Link, A.J., Eng, J., Schieltz, D.M., Carmack, E., Mize, G.J., Morris, D.R., Garvik, B.M., and Yates, J.R., 3rd (1999). Direct analysis of protein complexes using mass spectrometry. Nat Biotechnol 17, 676682.

MacLean, B., Tomazela, D.M., Shulman, N., Chambers, M., Finney, G.L., Frewen, B., Kern, R., Tabb, D.L., Liebler, D.C., and MacCoss, M.J. (2010). Skyline: an open source document editor for creating and analyzing targeted proteomics experiments. Bioinformatics 26, 966-968.

Malyutin, A.G., Musalgaonkar, S., Patchett, S., Frank, J., and Johnson, A.W. (2017). Nmd3 is a structural mimic of eIF5A, and activates the cpGTPase Lsg1 during 60S ribosome biogenesis. EMBO J.

Martin, I., Kim, J.W., Lee, B.D., Kang, H.C., Xu, J.C., Jia, H., Stankowski, J., Kim, M.S., Zhong, J., Kumar, M., et al. (2014). Ribosomal protein s15 phosphorylation mediates LRRK2 neurodegeneration in Parkinson's disease. Cell 157, 472-485.

Mauro, V.P., and Edelman, G.M. (2002). The ribosome filter hypothesis. Proceedings of the National Academy of Sciences of the United States of America 99, 12031-12036.

Mauro, V.P., and Edelman, G.M. (2007). The ribosome filter redux. Cell Cycle 6, 2246-2251.

Mazumder, B., Sampath, P., Seshadri, V., Maitra, R.K., DiCorleto, P.E., and Fox, P.L. (2003). Regulated release of L13a from the 60S ribosomal subunit as a mechanism of transcript-specific translational control. Cell 115, 187-198.

McIntosh, K.B., and Warner, J.R. (2007). Yeast Ribosomes: Variety Is the Spice of Life. Cell 131, 450451. 
Sun et al.

Identification of changing ribosomes using cryo-EM and MS

EMBL ver 2.8 (02/12/2018)

Mulder, A.M., Yoshioka, C., Beck, A.H., Bunner, A.E., Milligan, R.A., Potter, C.S., Carragher, B., and Williamson, J.R. (2010). Visualizing ribosome biogenesis: parallel assembly pathways for the 30S subunit. Science 330, 673-677.

Myasnikov, A.G., Afonina, Z.A., Menetret, J.F., Shirokov, V.A., Spirin, A.S., and Klaholz, B.P. (2014).

The molecular structure of the left-handed supra-molecular helix of eukaryotic polyribosomes. Nat Commun 5, 5294.

Nakao, A., Yoshihama, M., and Kenmochi, N. (2004). RPG: the Ribosomal Protein Gene database. Nucleic Acids Res 32, D168-170.

Pettersen, E.F., Goddard, T.D., Huang, C.C., Couch, G.S., Greenblatt, D.M., Meng, E.C., and Ferrin, T.E. (2004). UCSF Chimera--a visualization system for exploratory research and analysis. J Comput Chem $25,1605-1612$.

Pu, S., Vlasblom, J., Emili, A., Greenblatt, J., and Wodak, S.J. (2007). Identifying functional modules in the physical interactome of Saccharomyces cerevisiae. Proteomics 7, 944-960.

Ramagopal, S. (1992). Are eukaryotic ribosomes heterogeneous? Affirmations on the horizon. Biochem Cell Biol 70, 269-272.

Ramagopal, S., and Ennis, H.L. (1981). Regulation of synthesis of cell-specific ribosomal proteins during differentiation of Dictyostelium discoideum. Proc Natl Acad Sci U S A 78, 3083-3087.

Ray, P.S., Arif, A., and Fox, P.L. (2007). Macromolecular complexes as depots for releasable regulatory proteins. Trends in Biochemical Sciences 32, 158-164.

Ross, P.L., Huang, Y.L.N., Marchese, J.N., Williamson, B., Parker, K., Hattan, S., Khainovski, N., Pillai, S., Dey, S., Daniels, S., et al. (2004). Multiplexed protein quantitation in Saccharomyces cerevisiae using amine-reactive isobaric tagging reagents. Molecular \& Cellular Proteomics 3, 1154-1169.

Ruggero, D., and Pandolfi, P.P. (2003). Does the ribosome translate cancer? Nat Rev Cancer 3, 179-192. 
Sun et al.

Identification of changing ribosomes using cryo-EM and MS

EMBL ver 2.8 (02/12/2018)

Samir, P., Rahul, Slaughter, J.C., and Link, A.J. (2015). Environmental Interactions and Epistasis Are Revealed in the Proteomic Responses to Complex Stimuli. PLoS One 10, e0134099.

Scheres, S.H. (2012). RELION: implementation of a Bayesian approach to cryo-EM structure determination. J Struct Biol 180, 519-530.

Shen et al. in preparation.

Sherton, C.C., and Wool, I.G. (1974). A comparison of the proteins of rat skeletal muscle and liver ribosomes by two-dimensional polyacrylamide gel electrophoresis. Observations on the partition of proteins between ribosomal subunits and a description of two acidic proteins in the large subunit. J Biol Chem 249, 2258-2267.

Slavov, N., Semrau, S., Airoldi, E., Budnik, B., and van Oudenaarden, A. (2015). Differential Stoichiometry among Core Ribosomal Proteins. Cell Rep 13, 865-873.

Sonenberg, N., and Hinnebusch, A.G. (2009). Regulation of Translation Initiation in Eukaryotes: Mechanisms and Biological Targets. Cell 136, 731-745.

Sulima, S.O., Gulay, S.P., Anjos, M., Patchett, S., Meskauskas, A., Johnson, A.W., and Dinman, J.D. (2014). Eukaryotic rpL10 drives ribosomal rotation. Nucleic Acids Res 42, 2049-2063.

Suloway, C., Pulokas, J., Fellmann, D., Cheng, A., Guerra, F., Quispe, J., Stagg, S., Potter, C.S., and Carragher, B. (2005). Automated molecular microscopy: the new Leginon system. J Struct Biol 151, 41-60.

Unwin, R. D., Griffiths, J. R. and Whetton, A. D (2010). Simultaneous analysis of relative protein expression levels across multiple samples using iTRAQ isobaric tags with 2D nano LC-MS/MS. Nature Protocols 5, 1574-1582.

Warner, J.R. (1999). The economics of ribosome biosynthesis in yeast. Trends in Biochemical Sciences $24,437-440$.

Warner, J.R. (2015). Twenty years of ribosome assembly and ribosomopathies. RNA 21, 758-759. 
Sun et al.

Identification of changing ribosomes using cryo-EM and MS

EMBL ver $2.8(02 / 12 / 2018)$

Warner, J.R., and McIntosh, K.B. (2009). How common are extraribosomal functions of ribosomal proteins? Mol Cell 34, 3-11.

Winzeler, E.A., Shoemaker, D.D., Astromoff, A., Liang, H., Anderson, K., Andre, B., Bangham, R., Benito, R., Boeke, J.D., Bussey, H., et al. (1999). Functional characterization of the S. cerevisiae genome by gene deletion and parallel analysis. Science $285,901-906$.

Xue, S., Tian, S., Fujii, K., Kladwang, W., Das, R., and Barna, M. (2015). RNA regulons in Hox 5' UTRs confer ribosome specificity to gene regulation. Nature 517, 33-38.

Yates, J.R., Ruse, C.I., and Nakorchevsky, A. (2009). Proteomics by Mass Spectrometry: Approaches, Advances, and Applications. Annual Review of Biomedical Engineering 11, 49-79.

Zhou, X., Liao, W.J., Liao, J.M., Liao, P., and Lu, H. (2015). Ribosomal proteins: functions beyond the ribosome. J Mol Cell Biol 7, 92-104. 


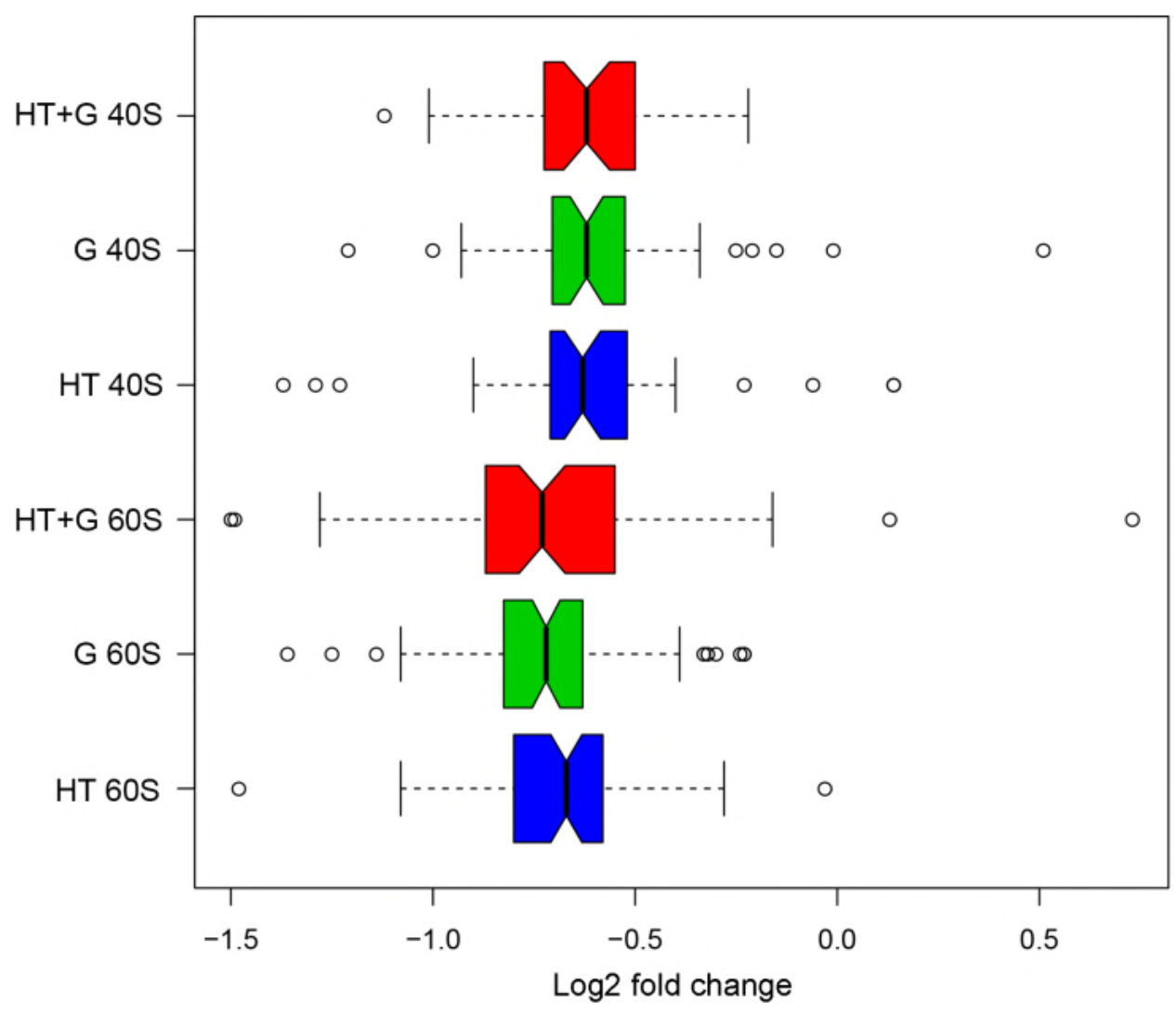

Fig. 1. The $\log _{2}$ fold-changes in the distributions of $40 \mathrm{~S}$ and $60 \mathrm{~S}$ subunit protein abundances caused by changes in growth conditions. Quantitative proteomics was used to identify and quantify changes in individual r-proteins abundances from $S$. cerevisiae whole-cell protein extracts after shifts in carbon source and temperature. Yeast was grown at standard conditions (glucose, $30^{\circ} \mathrm{C}$ ) and changed to three different environmental growth conditions or stimuli: high temperature $39^{\circ} \mathrm{C}$ (HT, blue); glycerol (G, green), and high temperature and glycerol combined (HT+G, red). A custom Python script CompZilla.py was used to analyze changes in the $r$-proteins in the quantitative proteomic data. Open circles represent $r$-protein outliers in the log ${ }_{2}$ fold-changes in their protein abundance. 
(A)
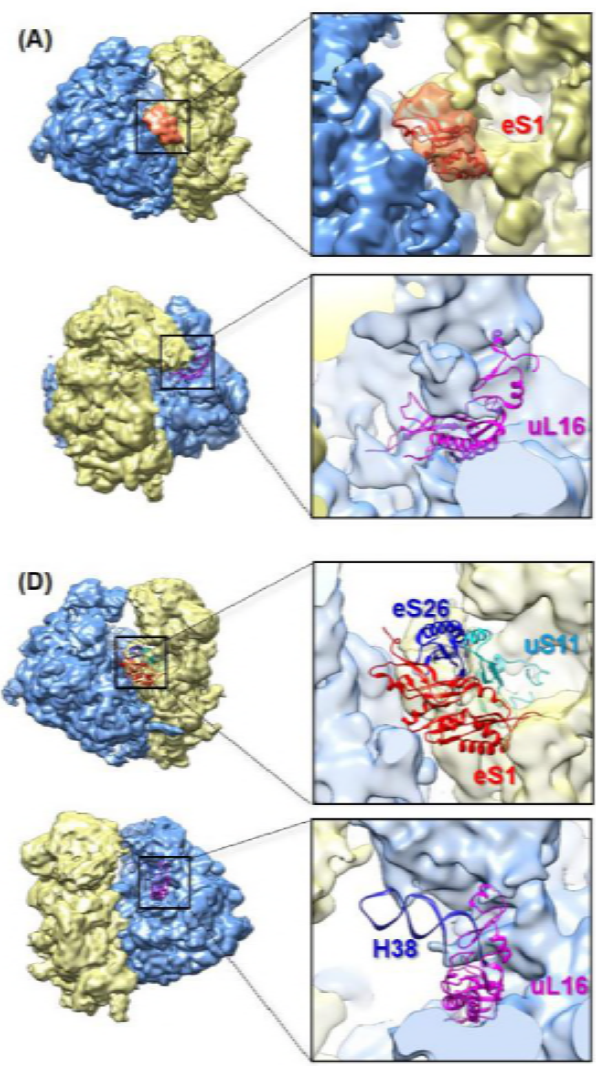

(B)
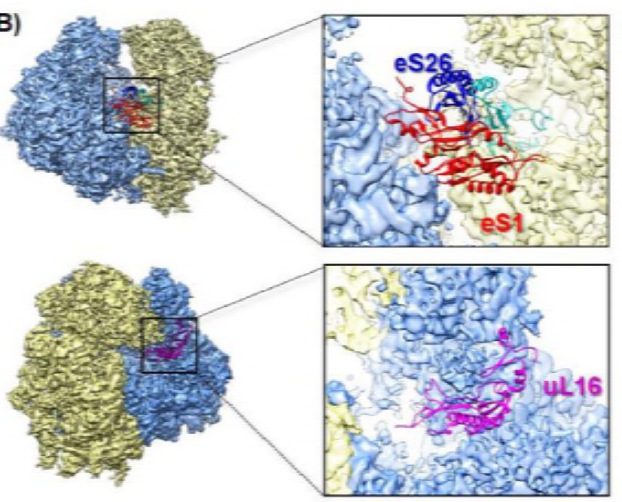

(E)
(C)
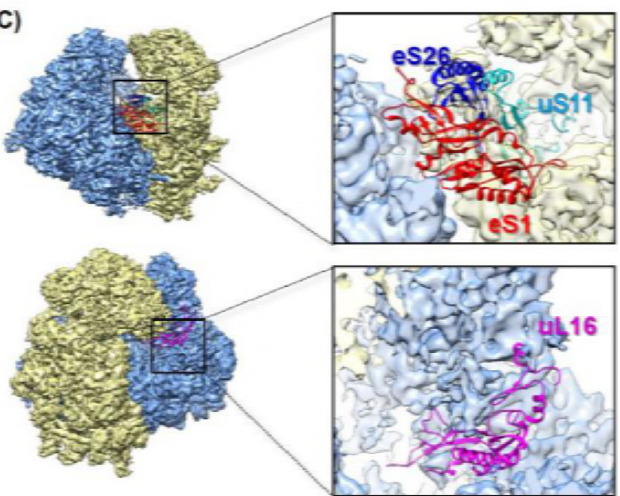
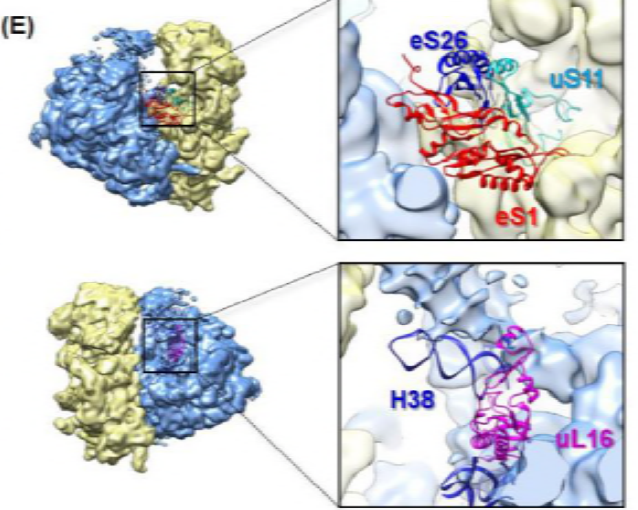

$60 S$ subunit
$40 S$ subunit
eS1 (4V88)
uL16 (4V88)
uS11 (4V88)
eS.26 (4V88)

Fig. 2. Cryo-EM reconstructions of $S$. cerevisiae $80 \mathrm{~S}$ ribosomes after the switch from glucose to glycerol. Yellow, $40 \mathrm{~S}$ subunit; blue, $60 \mathrm{~S}$ subunit. In all panels. A-E, two different views of the ribosome are shown, related by a $\sim 90$-degree rotation around a vertical axis in the plane of the figure. On the right hand side in each panel, a magnified slab of a region marked in the left view is shown, with densities shown in transparency. (A) Density map reconstructed from the sub-population of incomplete 80S ribosomes that lack only the density for RPL10 (uL16) protein but possess protein RPS1A/B (eS1) (orange). (B) Rotated incomplete $80 \mathrm{~S}$ ribosomes that lack the density for both RPS1A/B (eS1) and RPL10 (uL16) r-proteins, as well as the interacting partners of RPS1A/B (eS1),RPS26A/B (eS26), and RPS14A/B (uS11). (C) Non-rotated incomplete 80S ribosomes lacking the densities for both RPS1A/B (eS1) and RPL10 (uL16) r-proteins. (D, E) Incomplete 80 S ribosomes lacking densities for both the RPS1A/B (eS1) and RPL10 (uL16) proteins, as well as the H38 of the 28S rRNA. The interacting partners of RPS1A/B (eS1) (RPS26A/B (eS26) and RPS14A/B (US11)) are also missing entirely. The x-ray structures of S. cerevisiae (PDB 4V88) 40S and 60S subunits were rigid-body fitted separately into cryo-EM densities using UCSF Chimera (Pettersen et al., 2004). 


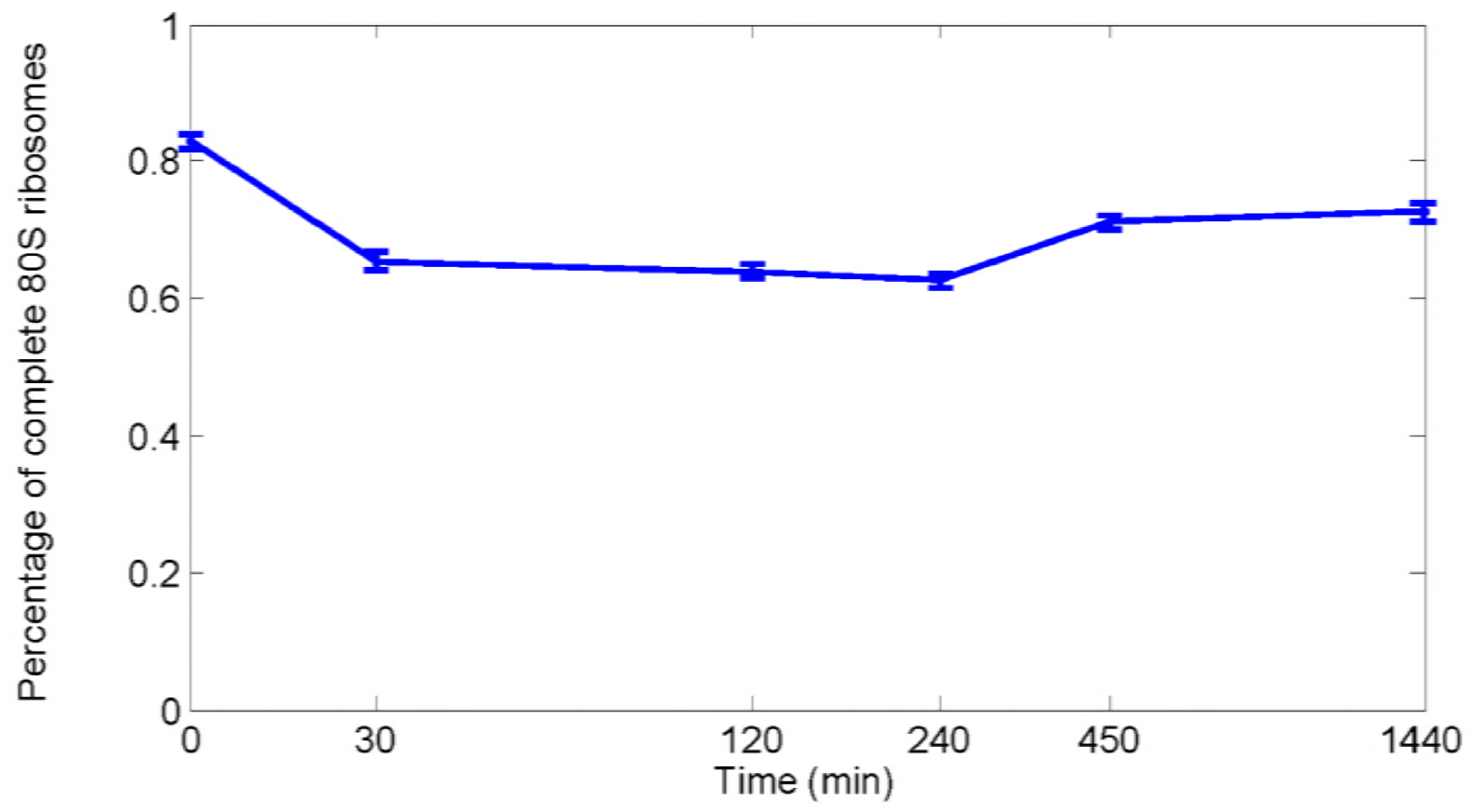

Fig. 3. Structural inventory as a function of time. The $x$-axis represents the time ( $\mathrm{min}, \log$ scale) that cells were incubated in glycerol medium. The $y$-axis represents the percentage of complete $80 \mathrm{~S}$ ribosomes. Generally, the percentage of complete $80 \mathrm{~S}$ ribosomes in each sample set decreased upon onset of the glucose-to-glycerol switch. This change reflects the general decrease in binding of RPS1A/B (eS1) and RPL10 (uL16) to 80S ribosomes. 
A

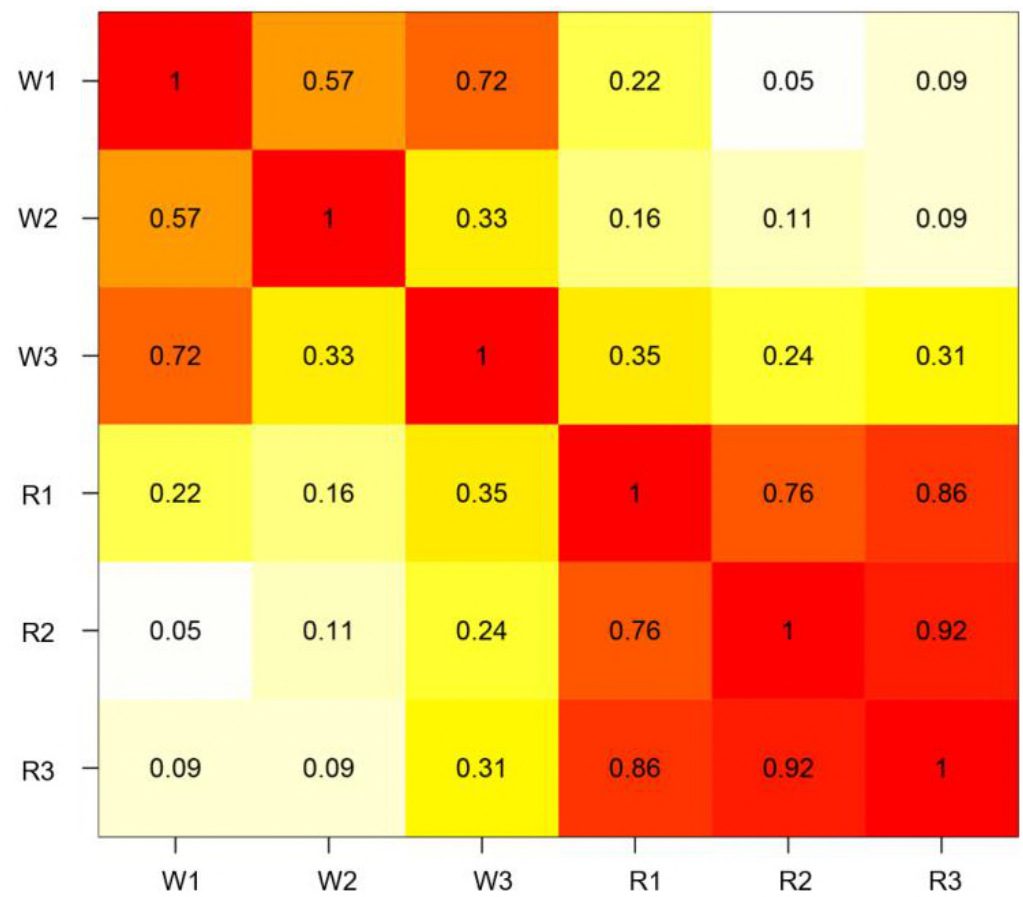

B

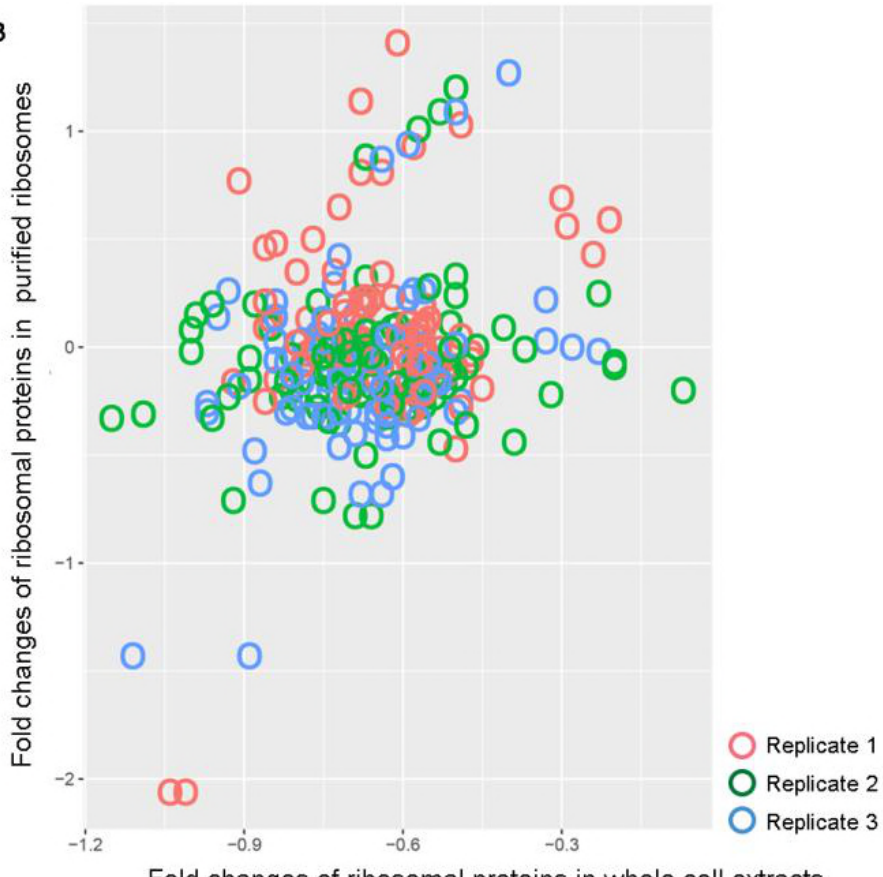

Fig. 4. Correlation analysis between the fold changes of r-proteins in whole cell extracts and purified ribosomes from cells grown at standard conditions (glucose, $\mathbf{3 0 ^ { \circ }} \mathrm{C}$ ) and glycerol (glycerol, $\mathbf{3 0}{ }^{\circ} \mathrm{C}$ ). (See Tables $\mathbf{S 2}$ and S3). The summary of three replicates is shown.

(A) Cross-correlation matrix. Numbers represent Pearson's R. W\# represent whole extract replicates. R\# represent purified ribosome replicates.

(B) Scatter plot showing the relationship between fold-changes in whole cell extracts ( $\mathrm{x}$-axis) and purified ribosomes ( $\mathrm{y}$-axis). 

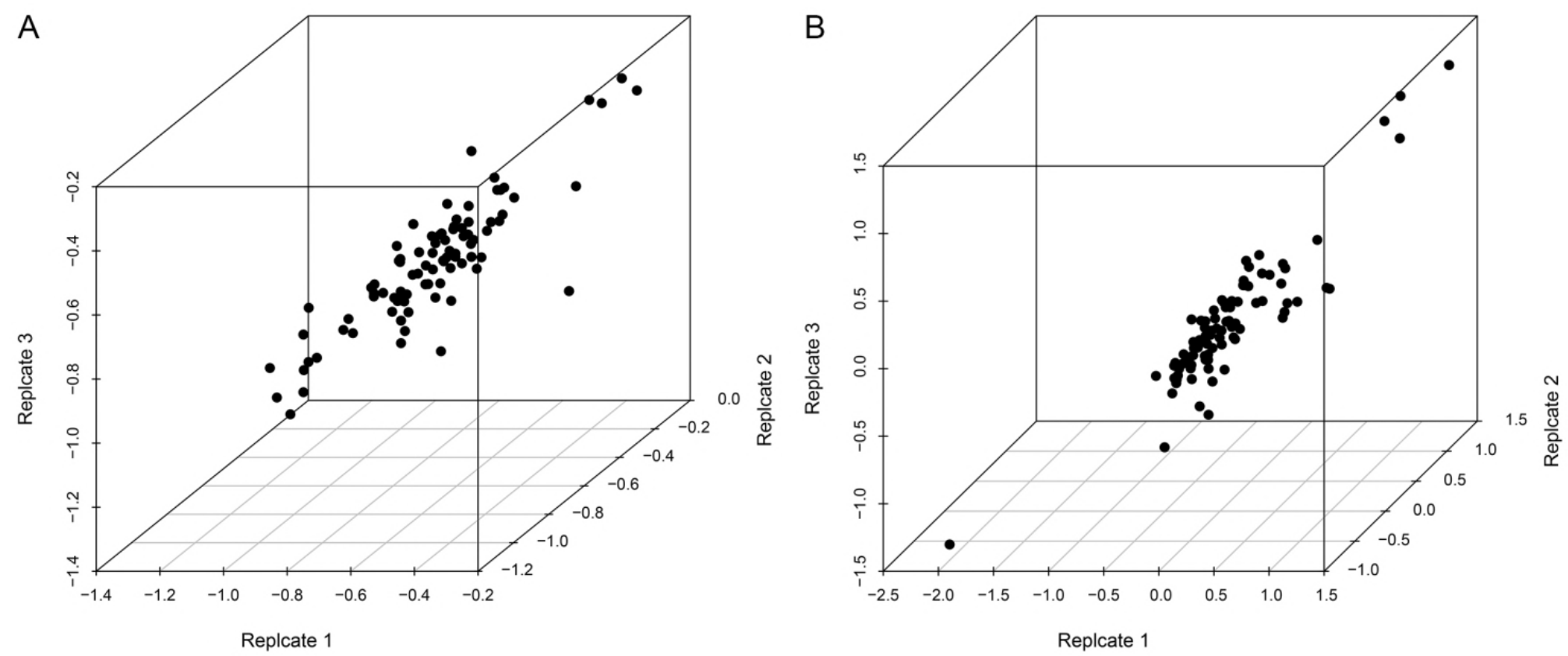

Fig. 5. Scatterplots showing reproducibility among replicates of whole cell extracts and purified ribosomes. (A) Whole-cell extract replicates. (B) Purified ribosome replicates. Plots were generated in RStudio. 
A

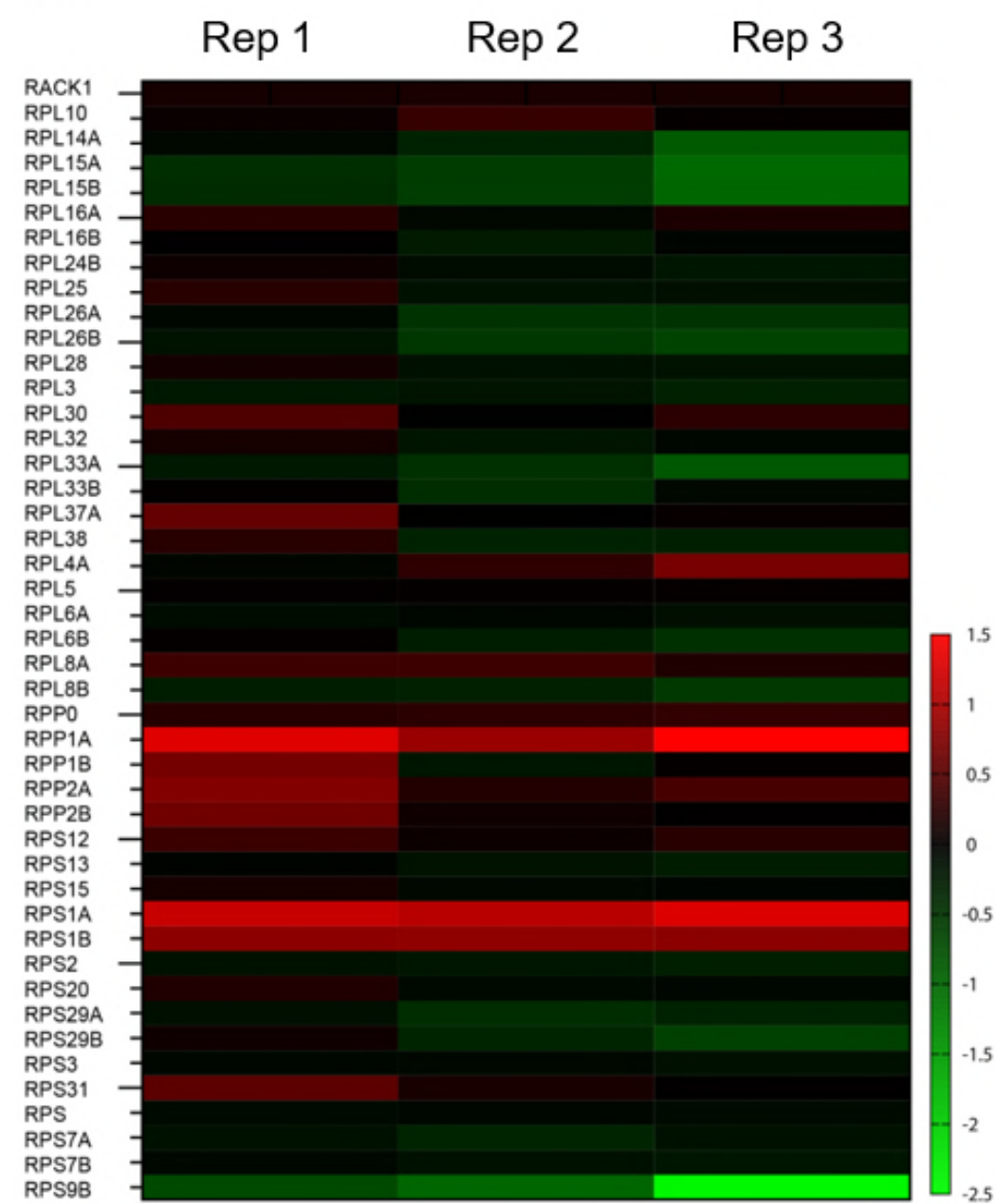

B

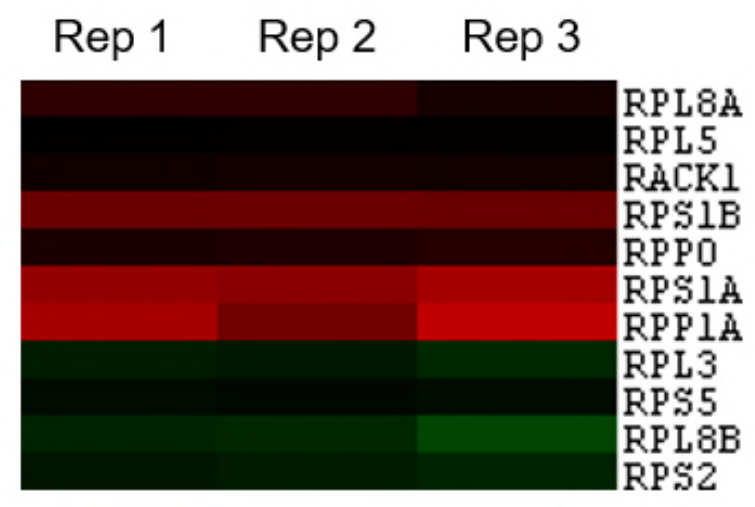

C

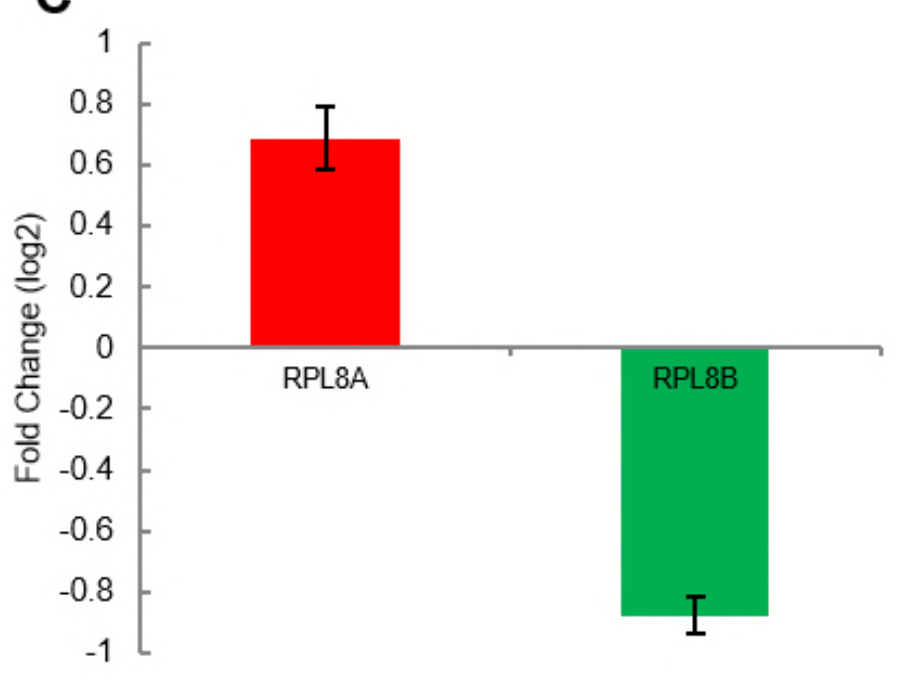

Fig. 6. Quantitation of r-proteins in ribosomes purified from yeast cells grown in glucose and glycerol. (A) Forty-five ribosomal proteins identified and quantitated using at least one unique peptide in iTRAQ-mass spectrometry experiments. The $\log _{2}$ transformed fold change in the r-protein's relative abundance $24 \mathrm{~h}$ after changing growth in glucose to glycerol was used to generate the heatmap. (B) Eleven statistically significant differentially abundant r-proteins in the purified ribosomes from cells grown in glucose and glycerol identified using a t-test's $p$-value less than 0.05 . (C) The protein abundance of RPL8A and RPL8B validated using multiple reaction monitoring in three independently purified ribosome replicates. Color Legend: red increased; green decreased abundance of the yeast $r$-protein in cells growing in glycerol compared to glucose media. 


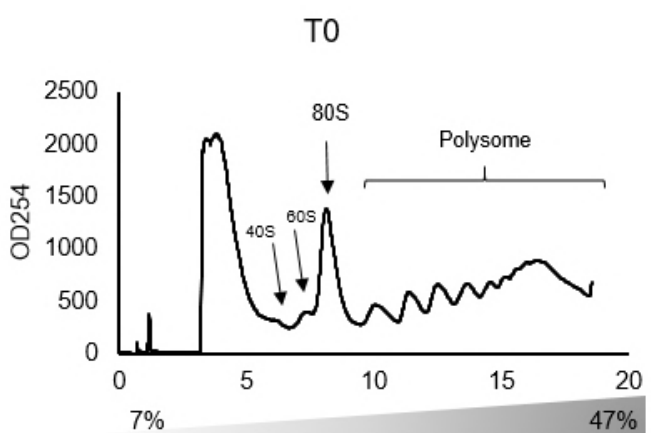

$\mathrm{T} 120$

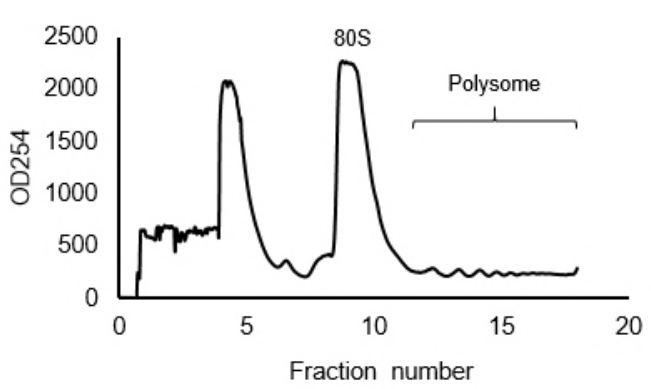

$\mathrm{T} 30$

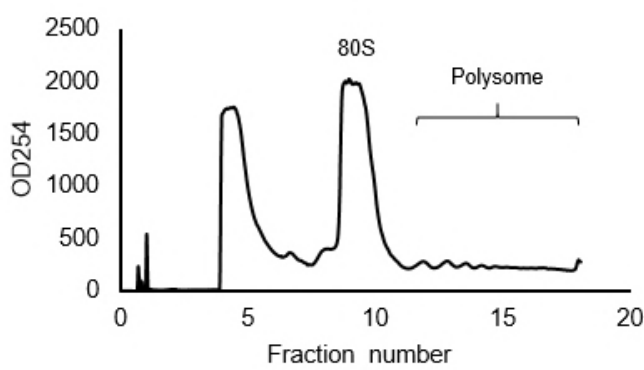

T240

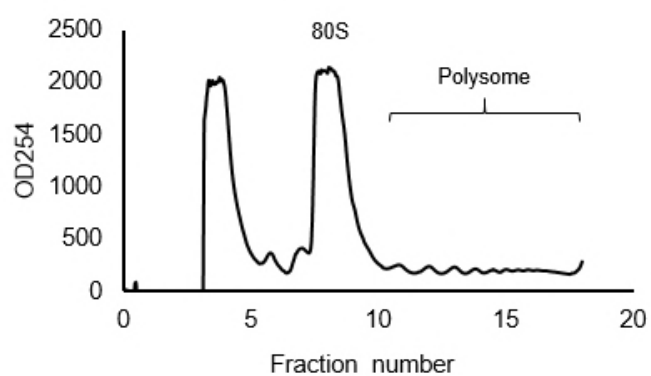

T60

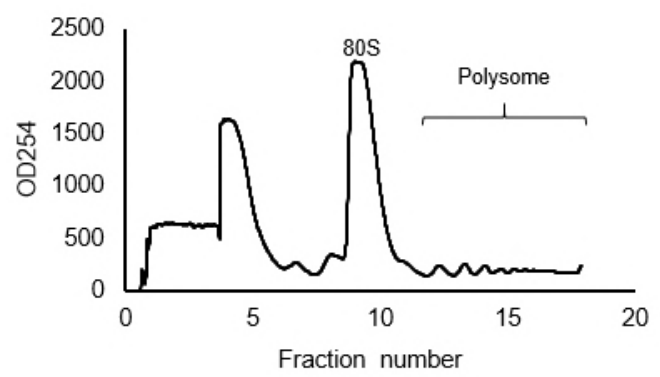

T450

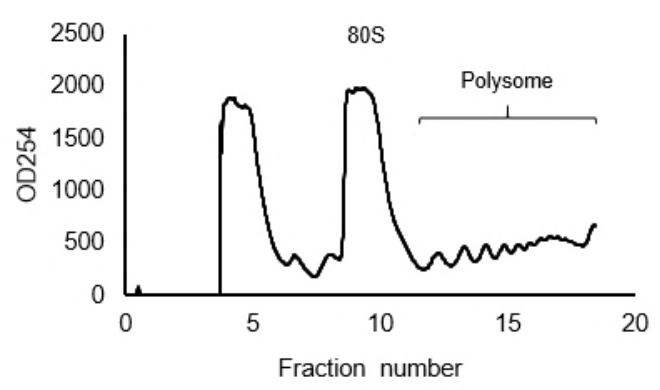

Fig. 7. The switch of carbon source from glucose to glycerol inhibits translation. Polyribosome traces from the wild-type S. cerevisiae strain. Yeast was grown in complete medium containing glucose, then re-suspended at $\mathrm{T}=0$ in medium containing glycerol for the indicated times (min). Polyribosomes were analyzed as described in 'STAR Methods'. The peaks that contain the small 40S ribosomal subunit, the large 60S ribosomal subunit, and complete $80 \mathrm{~S}$ ribosomes are indicated by arrows. The polysome peaks are bracketed. 

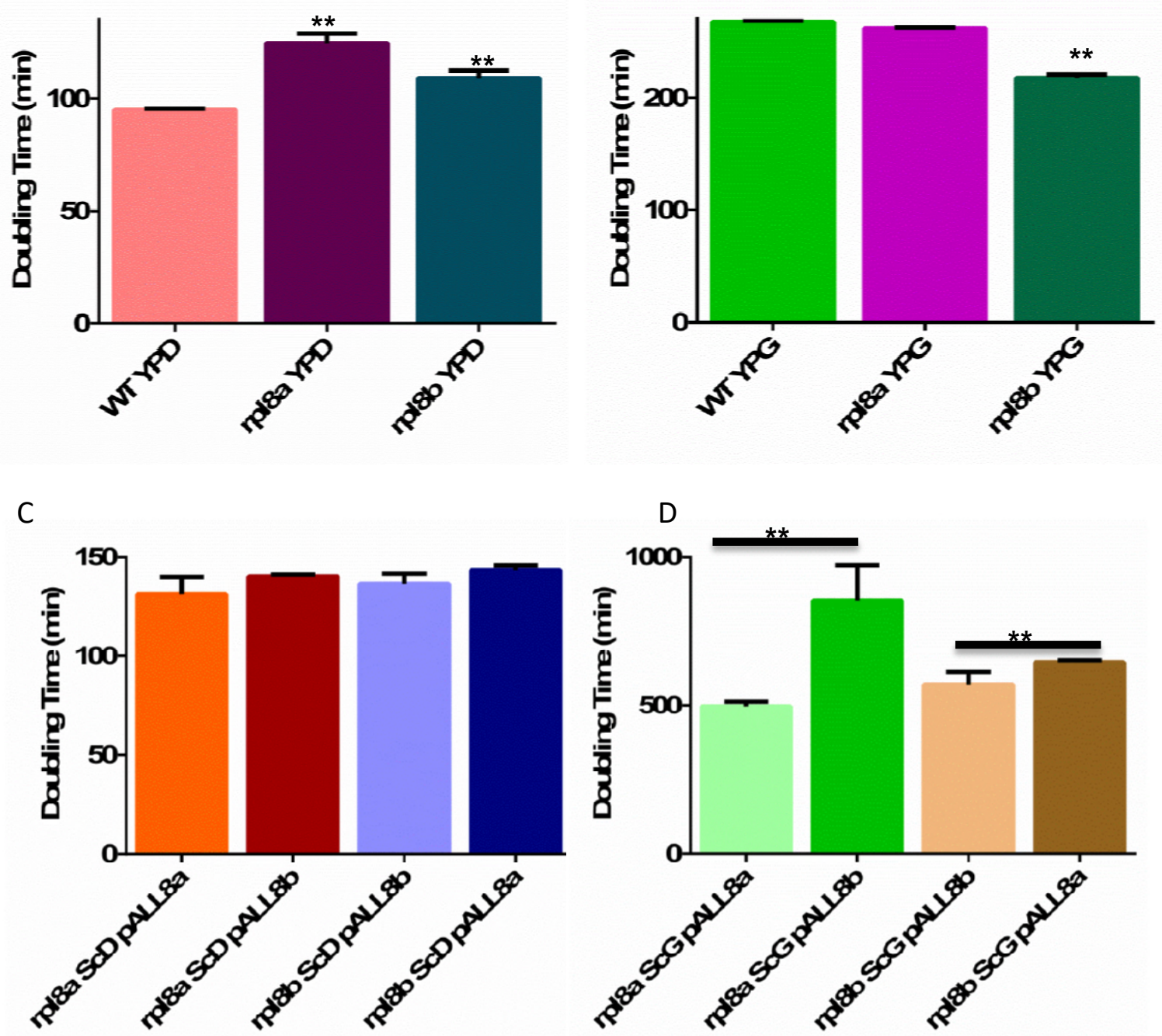

Fig. 8: Doubling times comparisons. A \& B) Stars denote the statistical significance of comparison with the WT strain ( $p$-values < 0.05$)$. A) Doubling times of WT, rp/8a, and rp/8b with glucose as carbon source. B) Doubling times of WT, $r p / 8 a$, and rpl $/ 8 b$ with glycerol as carbon source. C) Doubling times of $r p / 8 a$ with Rpl8a on plasmid, $r p / 8 a$ with Rpl8b on plasmid, $r p / 8 b$ with Rpl8b on plasmid, and $r p / 8 b$ with Rpl8a on plasmid with glucose as carbon source. C) Doubling times of $r p / 8 a$ with Rpl8a on plasmid, $r p / 8 a$ with Rpl8b on plasmid, $r p / 8 b$ with Rpl8b on plasmid, and $r p / 8 b$ with Rpl8a on plasmid with glycerol as carbon source. Stars denote the statistical significance of comparison denoted by the lines above the bar graph ( $p$-values < 0.05$)$. 


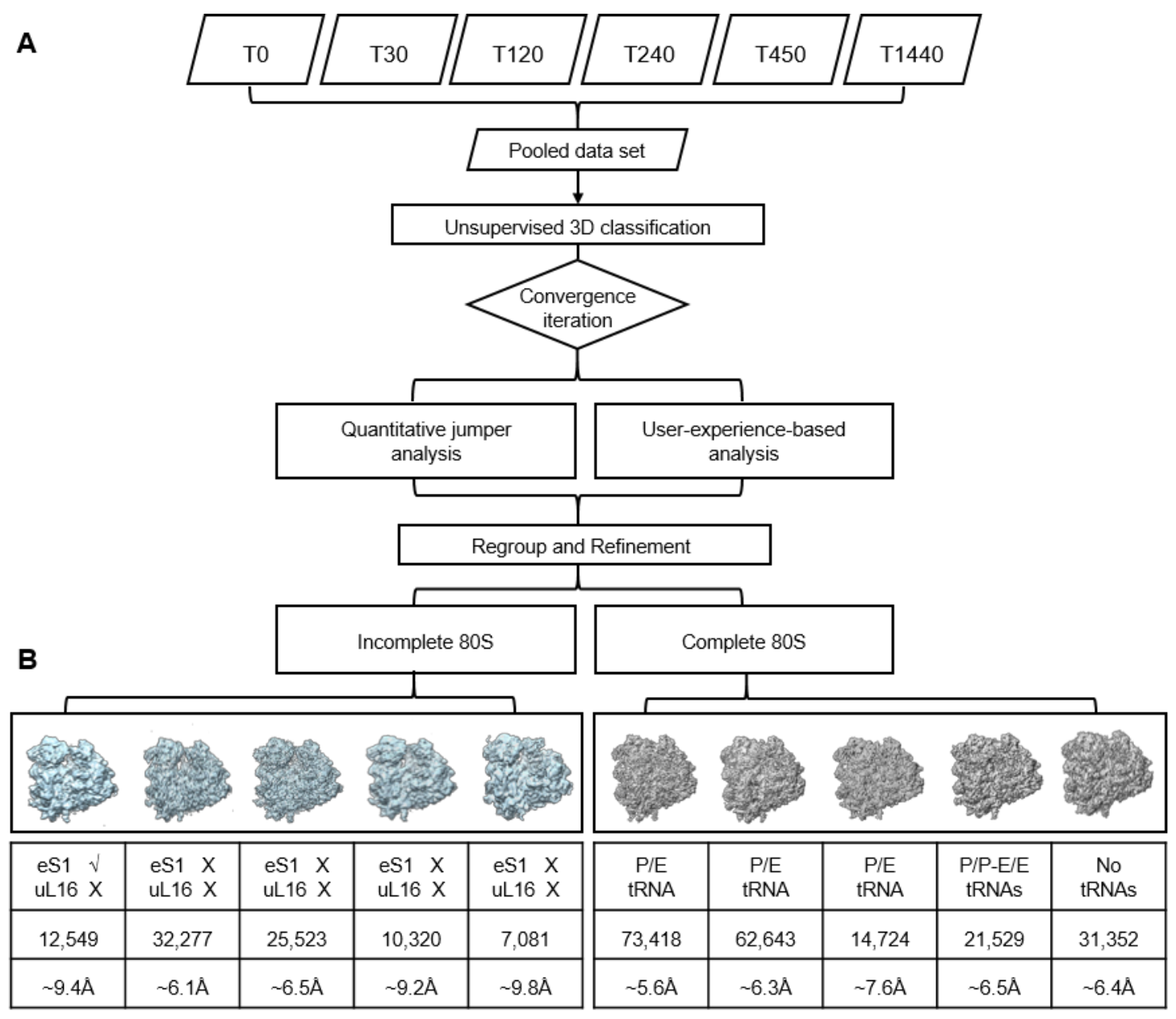

Fig S1. Full framework of 'pooled' classification strategy and results (Related to Fig 2). (A) Schematic diagram of our 'pooled classification' data processing procedure. Samples purified from six time points were pooled together and classified using the same procedure. Further analysis was based mainly on quantitative jumper analysis (20) with minimum user interference. (B) Summary of the full-scale cryo-EM studies. " $V$ ", present; " $X$ ", absent. Cryo-EM reconstructions of incomplete $80 \mathrm{~S}$ ribosomes are presented in front view and colored in light blue (B, left), while complete $80 \mathrm{~S}$ ribosomes are colored in grey (B, right). Resolutions reported are based throughout on the 'gold standard' protocol along with the FSC $=0.143 \mathrm{criterion}$, and involved soft masking and high-resolution noise substitution (Chen et al., 2013). 


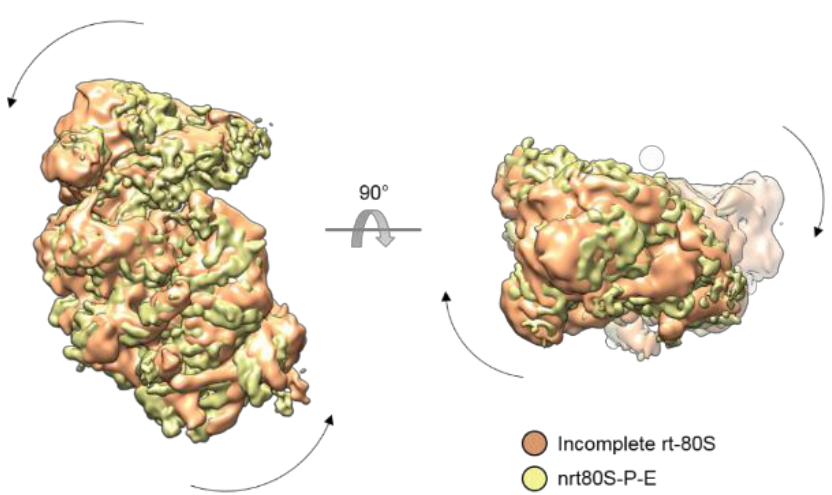

B

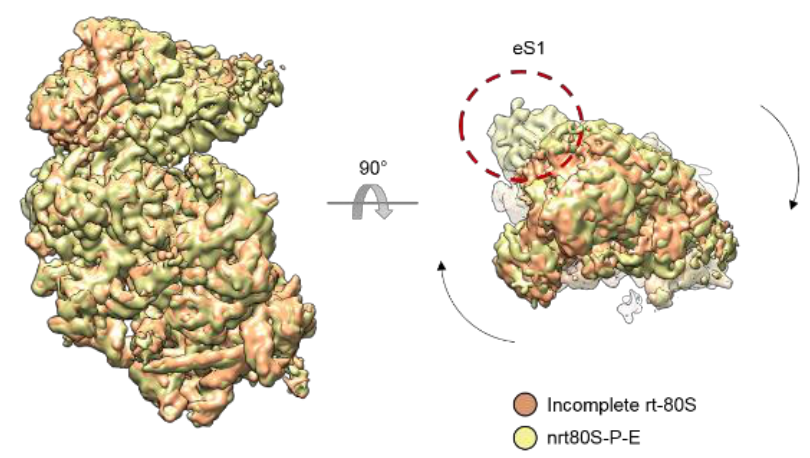

Figure S2. Global conformational changes of incomplete $80 \mathrm{~S}$ ribosomes (Related to Fig 2). (A) Inter-subunit rotation and 40S subunit head-swiveling movements in the incomplete $80 \mathrm{~S}$ (class 1). Comparison of the 40S subunit positions in nrt80S-P-E state (yellow) and the incomplete 80S (class 1) (orange). (B) Head-swiveling movements in the incomplete 80S (class 3). The position of eS1 on nrt80S-P-E state is highlighted by a red circle. Comparisons were obtained by structural alignment on the 60S subunits of the 80S ribosomes using UCSF Chimera (Pettersen et al., 2004). 
bioRxiv preprint doi: https://doi.org/10.1101/271833; this version posted February 26, 2018. The copyright holder for this preprint (which was not certified by peer review) is the author/funder, who has granted bioRxiv a license to display the preprint in perpetuity. It is made available under aCC-BY-NC-ND 4.0 International license.

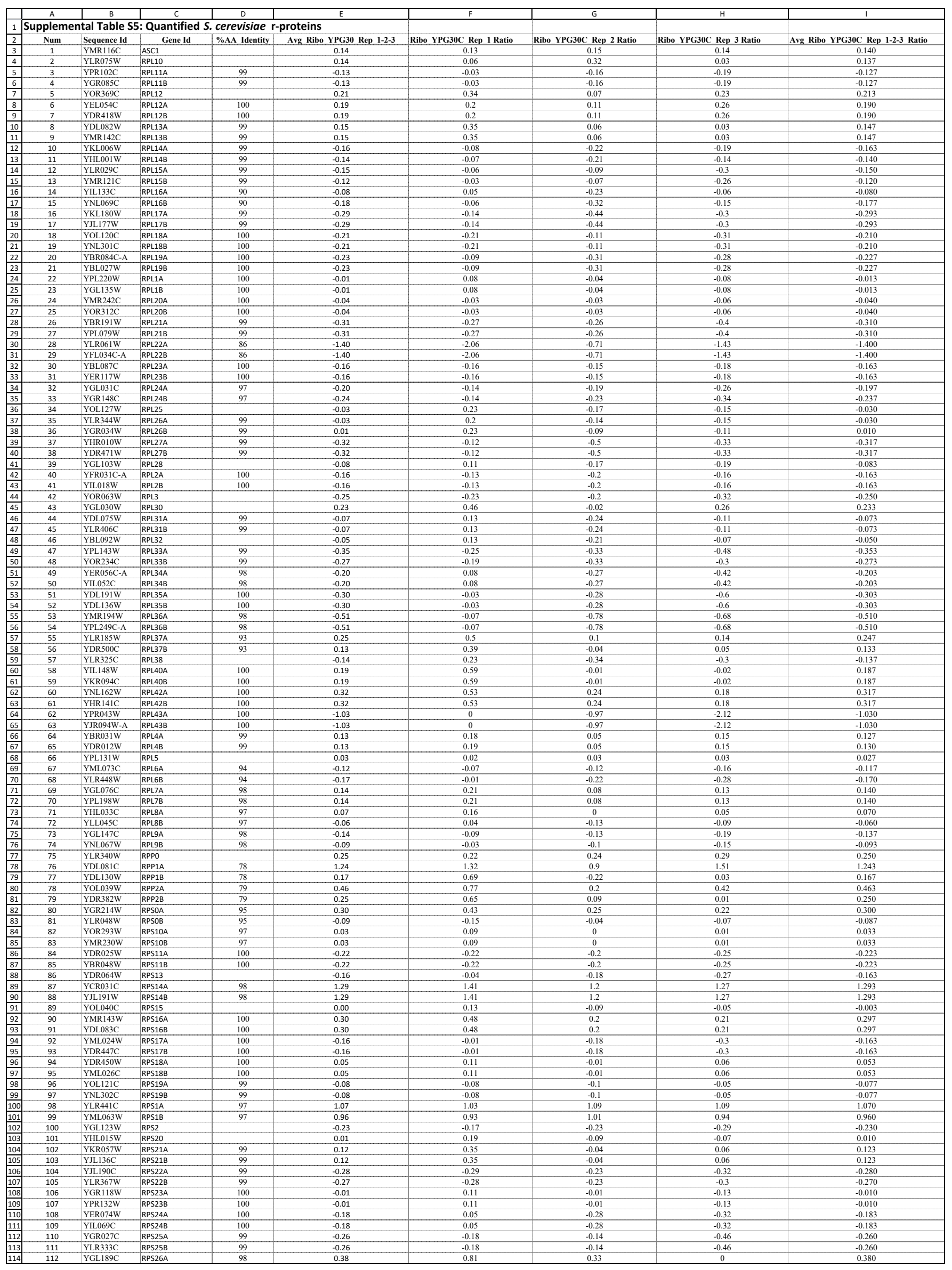


bioRxiv preprint doi: https://doi.org/10.1101/271833; this version posted February 26, 2018. The copyright holder for this preprint (which was not certified by peer review) is the author/funder, who has granted bioRxiv a license to display the preprint in perpetuity. It is made available under aCC-BY-NC-ND 4.0 International license.

\begin{tabular}{|c|c|c|c|c|c|c|c|c|c|}
\hline & A & B & C & D & $\mathrm{E}$ & $F$ & G & $\mathrm{H}$ & 1 \\
\hline 115 & 113 & YER131W & RPS26B & 98 & 0.37 & 0.81 & 0.28 & 0.03 & 0.373 \\
\hline 116 & 114 & YKL156W & RPS27A & 99 & -0.36 & -0.47 & -0.2 & -0.41 & -0.360 \\
\hline \begin{tabular}{|l|}
117 \\
\end{tabular} & 115 & YHR021C & RPS27B & 99 & -0.36 & -0.47 & -0.2 & -0.41 & -0.360 \\
\hline 118 & 116 & YLR388W & RPS29A & 91 & -0.31 & -0.15 & -0.44 & -0.33 & -0.307 \\
\hline 119 & 117 & YDL061C & RPS29B & 91 & -0.30 & 0.09 & -0.35 & -0.63 & -0.297 \\
\hline 120 & 118 & YNL178W & RPS3 & & -0.11 & -0.08 & -0.09 & -0.16 & -0.110 \\
\hline 121 & 119 & YLR287C-A & RPS30A & 100 & 0.96 & 1.14 & 0.88 & 0.87 & 0.963 \\
\hline 122 & 120 & YOR1 $182 \mathrm{C}$ & RPS30B & 100 & 0.96 & 1.14 & 0.88 & 0.87 & 0.963 \\
\hline 123 & 121 & YLR167W & RPS31 & & 0.22 & 0.56 & 0.09 & 0 & 0.217 \\
\hline 124 & 122 & YJR145C & RPSAA & 99 & -0.11 & -0.31 & 0.09 & -0.12 & -0.113 \\
\hline \begin{tabular}{|l|l|}
125 \\
\end{tabular} & 123 & YHR203C & RPS4B & 99 & -0.11 & -0.31 & 0.09 & -0.12 & -0.113 \\
\hline 126 & 124 & YJR123W & RPS5 & & -0.09 & -0.1 & -0.07 & -0.11 & -0.093 \\
\hline \begin{tabular}{|l|}
127 \\
\end{tabular} & 125 & YPL090C & RPS6A & 94 & -0.31 & -0.21 & -0.36 & -0.36 & -0.310 \\
\hline 128 & 126 & YBR181C & RPS6B & 94 & -0.31 & -0.21 & -0.36 & -0.36 & -0.310 \\
\hline \begin{tabular}{|l|}
129 \\
\end{tabular} & 127 & YOR096W & RPS7A & 98 & 0.03 & 0.05 & -0.04 & 0.07 & 0.027 \\
\hline 130 & 128 & YNL096C & RPS7B & 98 & 0.02 & 0.05 & 0 & 0.01 & 0.020 \\
\hline 131 & 129 & YBL072C & RPS8A & 97 & 0.20 & 0.12 & 0.21 & 0.26 & 0.197 \\
\hline 132 & 130 & YER102W & RPS8B & 97 & 0.20 & 0.12 & 0.21 & 0.26 & 0.197 \\
\hline 133 & 131 & YPL081W & RPS9A & 97 & 0.03 & 0 & 0.03 & 0.05 & 0.027 \\
\hline \begin{tabular}{|l|}
134 \\
\end{tabular} & 132 & YBR189W & RPS9B & 97 & -0.02 & -0.03 & -0.05 & 0.01 & -0.023 \\
\hline
\end{tabular}

